\title{
Quantum Dots for Improved Single-Molecule Localization Microscopy
}

Jennifer M. Urban ${ }^{\dagger}$, Wesley Chiang ${ }^{\ddagger}$, Jennetta W. Hammond ${ }^{\S}$, Nicole M. B. Cogan ${ }^{\dagger}$, Angela Stout ${ }^{\S}$ Rebeckah Burke ${ }^{\dagger}$, Harry A. Stern ${ }^{\#,}$ Harris A. Gelbard $\S^{*}$, Bradley L. Nilsson $^{+*}$, and Todd D. Krauss ${ }^{\dagger, £^{*}}$

${ }^{\dagger}$ Department of Chemistry, ${ }^{\ddagger}$ The Institute of Optics, and the \#Center for Integrated Research and Computing, University of Rochester, Rochester, New York 14627-0216, United States

${ }^{¥}$ Department of Biochemistry and Biophysics, ${ }^{\S}$ Center for Neurotherapeutics Discovery, Departments of Neurology, Pediatrics, Neuroscience and Microbiology and Immunology, University of Rochester Medical Center, Rochester, NY, 14642.

SUPPORTING INFORMATION

\section{Contents}

PEPTIDE SYNTHESIS . .53

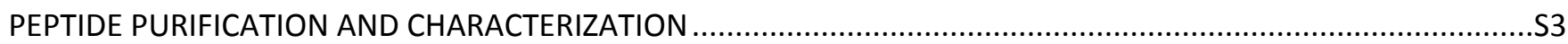

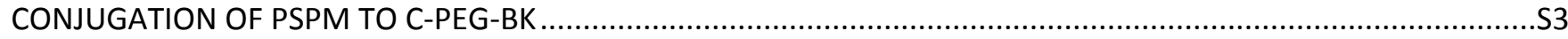

QUANTUM DOT SYNTHESIS: CdSe/CdS CORE/SHELL QDs FOR IMAGING ......................................................

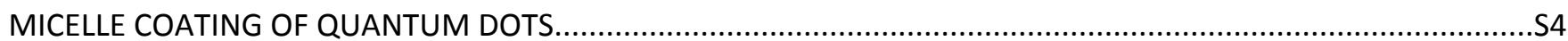

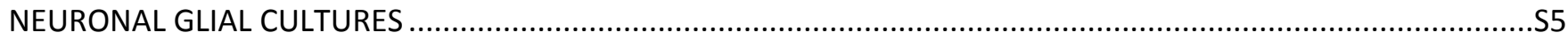

TREATING CULTURES WITH BKQDs AND/OR TAMRA-BK FOR SUPER-RESOLUTION IMAGING............................S5

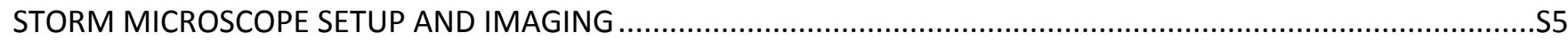

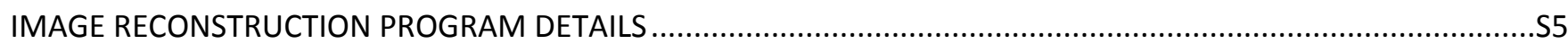

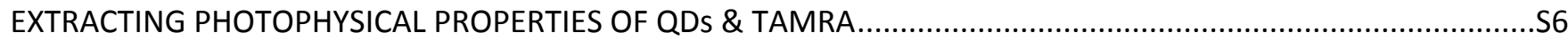

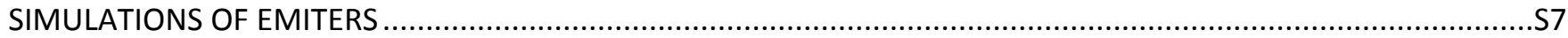

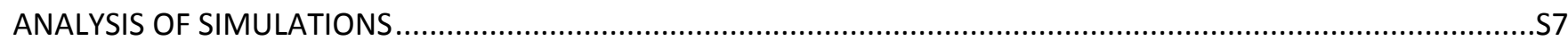

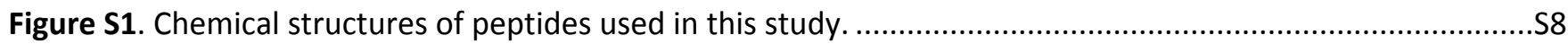

Figure S2. CdSe/CdS Characterization: Absorbance and Photoluminescence of CdSe Cores ..............................S8

Figure S3. CdSe/CdS Characterization: Absorbance and Photoluminescence of CdSe/CdS Core/Shell QDs...........S9

Figure S4. CdSe/CdS Characterization: TEM of CdSe/CdS Core/Shell QDs ......................................................S9

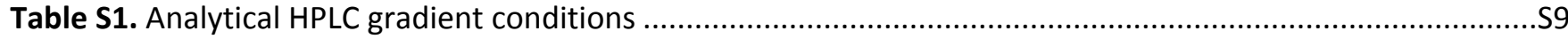

Figure S5. Analytical HPLC trace of synthetic peptide Bradykinin, BK, RPPGFSPFR-OH, at $215 \mathrm{~nm}$..................S10

Figure S6. Analytical HPLC trace of synthetic peptide C-PEG-Bradykinin, C-PEG-BK, Ac-C-PEG-RPPGFSPFR-OH, at $215 \mathrm{~nm}$

Figure S7. Analytical HPLC trace of synthetic peptide TAMRA-Bradykinin, TAMRA-BK, TAMRA-PEG-RPPGFSPFR-

$\mathrm{OH}$, at $215 \mathrm{~nm}$ 
Figure S8. Peptide concentration curves used to determine concentrations of all peptides except TAMRA-BK (for which UV-Vis was used) in this study: Bradykinin, BK, RPPGFSPFR-OH.

Figure S9. Peptide concentration curves used to determine concentrations of all peptides except TAMRA-BK (for which UV-Vis was used) in this study: C-PEG-Bradykinin, C-PEG-BK, Ac-C-PEG-RPPGFSPFR-OH. ..S11

Table S2. Calculated and observed $m / z$ for all peptides by MALDI-TOF-MS. ................................................S11

Figure S10. MALDI-TOF-MS spectrum for Bradykinin, BK, RPPGFSPFR-OH ..................................................S12

Figure S11. MALDI-TOF-MS spectrum for C-PEG-Bradykinin, C-PEG-BK, AC-C-PEG-RPPGFSPFR-OH...................S12

Figure S12. MALDI-TOF-MS spectrum for TAMRA-Bradykinin, TAMRA-BK, TAMRA-PEG-RPPGFSPFR-OH ...........S13

Figure S13. Diagram of pixel selection in ROI around bright, fluorescent spot..............................................S13

Figures S14. Representative time trace of BKQDs where the red-orange is the PL and the blue is the BG.........S13

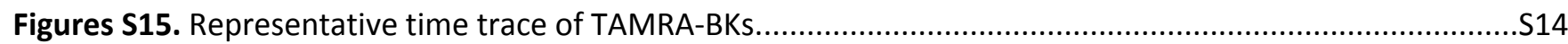

Figure S16. Background subtracted time trace, "PL-BG", and a smoothing kernel peak fit around the PDF of the

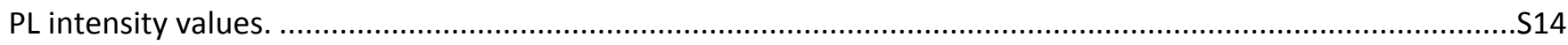

Figure S17. Changepoint trajectory (CP) fitted to "PL-BG" trace and subsequent "On-Off" transition blinking trace

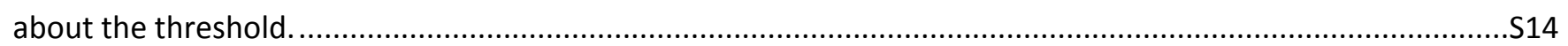

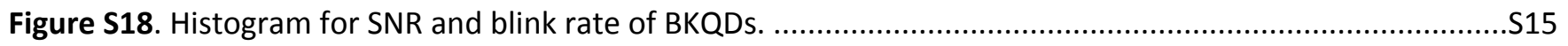

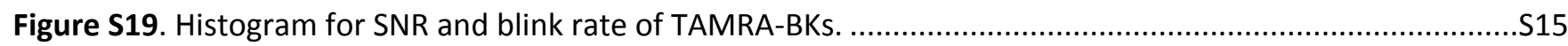

Figure S20. Creation of multi-frame movies of a simulated emitter........................................................S16

Figure S21. Diagram of how positions of the emitters are defined in the multi-emitter simulations to ensure substantial overlap.

Figure S22. Gaussian fitted histograms for the $X$ and $Y$ coordinates of the localized points from QuickPALM analysis of single emitter simulations.

Figure S23. Example hierarchical clustering analysis of localized points from QuickPALM analysis of multi-emitter simulations to identify groups of spots associated with one of the three simulated emitters.

Figure S24. Gaussian fitted histograms for the $X$ and $Y$ coordinates of the localized points from QuickPALM analysis of overlapped multiple emitter simulations. S18

Table S3. Results of centroid localization analysis for $X$ and $Y$ coordinates of single emitter simulations. . $\mathrm{S} 18$

Table S4. Results of centroid position analysis for 1000-frame multi-emitter simulations S19

Table S5. Results of centroid position analysis for 3000-frame multi-emitter simulations. . S19

Table S6. Comparison of QD photophysical properties to those reported in literature for well-performing, spectrally similar fluorophores normalized to TAMRA.....

Figure S25. Super-resolution imaging results of 30 day old samples showing the stability of fixed neuron samples treated with BKQDs over time.

Figure S26. Super-resolution imaging results of 30 day old samples showing the instability of TAMRA-BK treated, fixed neuron samples over time. .S20 


\section{PEPTIDE SYNTHESIS}

Canonical Fmoc- and sidechain-protected amino acids, Fmoc-8-amino-3,6-dioxaoctanoic acid (Fmoc-PEG), and 5-TAMRA NHS ester were purchased and used without further purification. Bradykinin, C-PEG-bradykinin, and TAMRA-PEG-bradykinin were synthesized using standard Fmoc-based solid-phase peptide synthesis on 100-200 mesh Wang acid resin pre-loaded with Fmoc-Arg(Pbf) (Novabiochem) using HBTU and HOBt activation for the majority of residues. DIC/HOBt activation was used for cysteine residues, HATU/HOAt activation was used for PEG residues, and HOAt alone for TAMRA coupling. Bradykinin was synthesized with free amine and carboxylic acid termini while the C-PEG- and TAMRA-PEG-variants were synthesized as $N$-acetyl, $C$-terminal acid sequences. After Fmoc deprotection of the final amino acid and $\mathrm{N}$-acetylation as appropriate, side chain deprotection and cleavage from the resin was performed using a 95:2.5:2.5 v/v/v trifluoroacetic acid:triisopropylsilane:water solution. The resulting solution was concentrated and precipitation initiated via addition of cold diethyl ether. The precipitate was collected via centrifugation and dissolved in a 60:40 acetonitrile:water mixture prior to purification by preparatory HPLC.

\section{PEPTIDE PURIFICATION AND CHARACTERIZATION}

Peptide purification was performed using a reverse phase Phenomenex Gemini $(5 \mu \mathrm{m}, \mathrm{NX}-\mathrm{C} 18,110 \AA$, $250 \times 50$ $\mathrm{mm}$ ) column on an Interchim Puriflash 4125 preparatory HPLC with a binary gradient of water and acetonitrile with $0.1 \%$ TFA at $100 \mathrm{~mL} \mathrm{~min}^{-1}$. For fraction collection, UV absorbance of the eluent was monitored at 215 and $254 \mathrm{~nm}$. Peptide purity was confirmed using analytical HPLC with a reverse phase Phenomenex Gemini $(5 \mu \mathrm{m}$, C18, $110 \AA, 250 \times 4.6 \mathrm{~mm}$ ) column on a Shimadzu LC-2010A and via MALDI-TOF mass spectroscopy. Peptide concentration for all experiments was determined by analyzing samples via analytical HPLC for comparison to a concentration curve calibrated by amino acid analysis. Appropriate amounts were then aliquoted and lyophilized prior to use.

\section{CONJUGATION OF PSPM TO C-PEG-BK}

Phosphatidylserine-PEG-maleimide (PSPM) was purchased from Nanocs Inc (PG2-MLPS-2k) and C-PEGBradykinin was synthesized in house as described above. A 1.6× excess of C-PEG-Bradykinin compared to PSPM was dissolved in degassed $1 \times$ PBS. A 100x excess of TCEP to peptide was added and the solution stirred under nitrogen for 20 minutes to reduce any disulfide bonds. PSPM in $1 \times$ PBS was then added and the solution stirred under nitrogen overnight. To remove TCEP, unreacted C-PEG-Bradykinin, and buffer salts, the reaction solution was transferred to a Slide-A-Lyzer Dialysis Cassette G2 with a 2,000 molecular weight cutoff (Thermo Scientific) and dialyzed against fresh nanopure water for 2 hours, twice, and then overnight. After dialysis, the solution was removed from the cassette and lyophilized to give a dry powder of Bradykinin-tagged phosphatidylserine.

\section{QUANTUM DOT SYNTHESIS: CDSE/CDS CORE/SHELL QDS FOR IMAGING}

Synthesis method was adapted from Kress et. al. ${ }^{1}$ Prior to synthesis, $1 \mathrm{M}$ trioctylphosphine-selenide (TOPSe) was made by mixing $680 \mathrm{mg}$ Se pellets and $8.5 \mathrm{~mL}$ trioctylphosphine (TOP) until clear in a glovebox at $60^{\circ} \mathrm{C}$ overnight. Then, $90 \mu \mathrm{L}$ diphenyl phosphine (DPP) was added to this solution.

CdSe cores were synthesized by first adding $820 \mathrm{mg} \mathrm{CdO}, 16.2 \mathrm{~g}$ trioctylphosphine oxide (TOPO), $37 \mathrm{~g}$ hexadecylamine (HDA), and $3.2 \mathrm{~g} \mathrm{n}$-dodecylphosphonic acid (DDPA) to a $250 \mathrm{~mL}$ flask. The flask was heated to $90^{\circ} \mathrm{C}$ under $\mathrm{N}_{2}$ and degassed by evacuating below $100 \mathrm{mT}$ and refilling with $\mathrm{N}_{2}$ three times. Under $\mathrm{N}_{2}$ atmosphere, the flask was heated to $320^{\circ} \mathrm{C}$ with rapid stirring until the solution was completely clear, indicating complexation of Cd-DDPA. The flask was then cooled to $260^{\circ} \mathrm{C}$, and $8.0 \mathrm{~mL}$ TOPSe was rapidly injected while stirring. The QDs were grown for 3 hours until the desired size was achieved. To quench the reaction, the heating mantle was removed and forced air was used to cool the flask to $200^{\circ} \mathrm{C}$. A water bath was used to further cool the reaction to 
$100{ }^{\circ} \mathrm{C}$. Next, $40 \mathrm{~mL}$ butanol was added to the flask, and the solution was left to cool for one hour. The solution was then divided between six $50 \mathrm{~mL}$ falcon tubes. Each solution was diluted to $50 \mathrm{~mL}$ with methanol and centrifuged for 10 minutes. The clear supernatant was discarded. After the QD pellets were allowed to dry for 15 minutes, the QDs were re-dispersed in $20 \mathrm{~mL}$ hexane, each, and left overnight. The following day, the QDs were centrifuged for 20 minutes, and the colored supernatant was transferred to 6 fresh $50 \mathrm{~mL}$ tubes, containing about $25 \mathrm{~mL}$ of solution in each tube. To wash the QDs, samples were precipitated with ethanol and resuspended in hexane twice.

The resulting CdSe core stock was purified via two cycles of size-selective precipitation with ethanol. The final QD pellet was dissolved in hexane and filtered through a $0.45 \mu \mathrm{m}$ syringe filter. This filtered solution was then used for the shelling reaction. Prior to shelling, Cd-oleate was made by placing $250 \mathrm{mg} \mathrm{CdO}, 2.6 \mathrm{~mL}$ oleic acid, and $20 \mathrm{~mL}$ octadecylamine in a flask, which was degassed at room temperature. Then, under $\mathrm{N}_{2}$, the flask was heated to $270^{\circ} \mathrm{C}$ for $90 \mathrm{~min}$ until clear. The flask was then cooled to $150^{\circ} \mathrm{C}$, and $1.3 \mathrm{~mL}$ oleylamine was added. The Cdoleate was transferred to the glovebox and stored until use.

To shell the CdSe cores, $3 \mathrm{~mL}$ of oleylamine, $3 \mathrm{~mL}$ of octadecene, and $2.2 \mathrm{~mL}$ of the CdSe core stock solution were stirred at $500 \mathrm{rpm}$ in a $100 \mathrm{ml}$ flask and degassed at room temperature for 70 minutes to remove the hexanes. The flask was then heated to $115^{\circ} \mathrm{C}$, degassed for 20 minutes, and refilled with $\mathrm{N}_{2}$. Meanwhile, the $\mathrm{Cd}$ and $\mathrm{S}$ precursors were prepared in the glovebox. $2.26 \mathrm{~mL}$ of the previously prepared Cd-oleate was diluted to $3.5 \mathrm{~mL}$ with octadecene and loaded into a $5 \mathrm{~mL}$ syringe. For the $\mathrm{S}$ precursor, $0.04 \mathrm{~mL}$ octanethiol was also diluted in 3.5 $\mathrm{mL}$ octadecene and added to a second $5 \mathrm{~mL}$ syringe. The syringes were removed from the glovebox and affixed to a syringe pump, set to a rate of $1.5 \mathrm{~mL}$ per hour. Next, the heating mantle was set to a temperature ramp of $16^{\circ} \mathrm{C}$ per minute. At $200^{\circ} \mathrm{C}$, precursor infusion was initiated, and once the flask reached $305^{\circ} \mathrm{C}$, the reaction temperature was maintained for two hours for shell growth. Then, the flask was cooled to $200^{\circ} \mathrm{C}, 1 \mathrm{~mL}$ oleic acid was added dropwise, and the solution was left to anneal for one hour. Once cooled to $75^{\circ} \mathrm{C}$, the reaction mixture $(20 \mathrm{~mL})$ was transferred to a $50 \mathrm{~mL}$ tube, diluted to $50 \mathrm{~mL}$ with ethanol, and centrifuged for 15 minutes. The supernatant was discarded, and the QD pellet was allowed to dry. Next, the pellet was re-dissolved in $5 \mathrm{~mL}$ hexane, diluted to $35 \mathrm{~mL}$ with ethanol, and centrifuged for 15 minutes. Then, the supernatant was again discarded, and the pellet was allowed to dry. Finally, the resulting pellet was dissolved in $6 \mathrm{~mL}$ hexane and centrifuged for 5 minutes. The supernatant containing $\mathrm{CdSe} / \mathrm{CdS}$ QDs was collected and stored in the glovebox until the QDs were required for experiments.

\section{MICELLE COATING OF QUANTUM DOTS}

$\mathrm{CdSe} / \mathrm{CdS}$ core/shell quantum dots passivated with oleic acid in hexane with an emission maximum at $627 \mathrm{~nm}$ were synthesized by Kelly Sowers and Rebecca Burke of the Krauss group at the University of Rochester. The dots were measured to be 5-6 nm in diameter via TEM and UV-Vis analysis. Lipid-PEG (1,2-distearoyl-sn-glycero-3phosphoethanolamine- $\mathrm{N}$-[methoxy(polyethylene glycol)-2000] (ammonium salt); product number 880120) in chloroform was purchased from Avanti Polar Lipids. QDs were coated with micelles composed of lipid-PEG and phosphatidylserine-bradykinin (PSPM-BK) using a modified procedure from Maiseyeu et al. ${ }^{2}$. Lipid-PEG and PSPMBK were first mixed in chloroform in a 40:60 $\mathrm{m} / \mathrm{m}$ ratio. $0.0075 \mathrm{~nm}$ QDs in chloroform were then added and the solution sonicated for 20 minutes to break up any pre-formed micelles. Next, the mixture was dried to a film under a gentle stream of nitrogen and re-dissolved in nanopure water to give a final concentration of $50 \mathrm{nM}$ QDs. This solution was vortexed for 1 minute and then sonicated 20 minutes to facilitate incorporation of QDs into micelles. The micelle-coated QDs were purified using Illustra MicroSpin S-300 HR Columns from GE Healthcare following the suggested protocol, with the resulting eluent collected for use in cell culture treatments.

\section{NEURONAL GLIAL CULTURES}


Primary hippocampal cultures were prepared from embryonic day 18 (E18) Sprague Dawley rats. Animal care and use were carried out in accordance with the recommendations of the Guide for the Care and Use of Laboratory Animals and protocols were approved by the University Committee on Animal Resources at the University of Rochester. Hippocampi were dissected out and dissociated in $0.25 \%$ trypsin. Cells were plated onto poly-D-lysine coated coverslips or culture dishes in neurobasal media supplemented with B27 with antioxidants, GlutaMAX, 5\% FBS, and $25 \mu \mathrm{M}$ glutamic acid. Cells were fed every 3-4 days with neurobasal media supplemented with B27 without antioxidants and GlutaMAX. At 7 days in vitro (DIV), cells were treated with the anti-mitotics 5-fluorodeoxyuridine (fUDR) at $20 \mathrm{mg} / \mathrm{mL}$ and uridine at $50 \mathrm{mg} / \mathrm{mL}$ to limit glial growth.

\section{TREATING CULTURES WITH BKQDS AND/OR TAMRA-BK FOR SUPER-RESOLUTION IMAGING}

For BKQD or PEGQD treatment, $50 \mathrm{nM}$ QD solution in water was diluted to $1 \mathrm{nM}$ in neurobasal media with GlutaMAX and B27 with antioxidants and mixed well. Next, media in wells was removed via aspiration and replaced with $2 \mathrm{~mL} \mathrm{QD/media} \mathrm{solution} \mathrm{per} \mathrm{well} \mathrm{and} \mathrm{then} \mathrm{incubated} \mathrm{at} 37^{\circ} \mathrm{C}$ for 15 minutes. After incubation, $\mathrm{QD} /$ media solution was removed from wells via aspiration and washed with $2 \mathrm{~mL}$ neurobasal media (with glutathione and antioxidants) for 5 minutes. Cells were fixed with 4\% paraformaldehyde (PFA) in PBS for 10 minutes, then incubated with $100 \mathrm{mM}$ glycine in PBS for 5 minutes, and washed twice with PBS. Coverslips were stored in $1 \times$ PBS and imaged the same day as they were prepared.

For TAMRA-BK treatment, a $2500 \mathrm{nM}$ solution of TAMRA-BK in water was diluted to $500 \mathrm{nM}$ in neurobasal media with GlutaMAX and B27 with antioxidants and mixed well. A large excess of TAMRA-BK was used in comparison to BKQD treatments in an attempt to approximate the same amount of BK in each sample (knowing that there are many BK molecules per QD). All treatment steps after this point are the same as for QDs.

\section{STORM MICROSCOPE SETUP AND IMAGING}

All single molecule imaging experiments were performed on a Nikon Eclipse TE300 inverted microscope configured for total internal reflection fluorescence (TIRF) with a Nikon Apo TIRF 60x, 1.49 NA oil objective. Samples were excited with continuous illumination from a $568 \mathrm{~nm}$ Spectra-Physics Millennia diode-pumped laser with output power adjusted to $12 \mathrm{~mW}$ for QDs or $50 \mathrm{~mW}$ for TAMRA using both adjustment of the laser power supply and neutral density filters. When imaging QDs, $594 \mathrm{~nm}$ and $568 \mathrm{~nm}$ long pass emission filters were used to remove excess excitation light. When imaging TAMRA, two $532 \mathrm{~nm}$ long pass emission filters were used. Movies were recorded using a scientific complementary metal oxide semiconductor (sCMOS) pco.edge 4.2 camera. All single-molecule imaging experiments involved collecting 1,000 frame movies with $25 \mathrm{~ms}$ exposure per frame.

\section{IMAGE RECONSTRUCTION PROGRAM DETAILS}

The ImageJ plugin QuickPALM was used to provide the location of all spots that met a chosen minimum signalto-noise cutoff. ${ }^{3}$ Next, the results were run through a custom Python script "csv2png." This script takes as input a csv-formatted text file output from QuickPALM, and plots the localized spots in PNG image format. The center of each supporting is plotted as an opaque point at the mean position of points assigned to a spot, surrounded by a semi-transparent circle with a radius equal to the localization precision $\sigma$, calculated by eq $1 .{ }^{4}$

$$
\sigma=\sqrt{\frac{s^{2}}{N}+\frac{a}{12 N}+\frac{8 \pi s^{4} b^{2}}{a^{2} N^{2}}}
$$

Here $\mathrm{N}$ is the intensity (the number of points assigned to a spot), $\mathrm{b}$ is the standard deviation of the background intensity, $a$ is the pixel size, and $s$ is the standard deviation of point positions. Assuming an isotropic Gaussian distribution, s was estimated from the four mean absolute deviations describing spot shape given by QuickPALM via eq 2 : 


$$
S=\sqrt{\frac{\pi}{2}}\left[\frac{\sigma_{l}+\sigma_{r}+\sigma_{u}+\sigma_{d}}{4}\right]
$$

\section{EXTRACTING PHOTOPHYSICAL PROPERTIES OF QDS \& TAMRA}

The combined output of QuickPALM and the "csv2png" script were used to identify locations of potential single emitters in the movies of BKQDs and TAMRA-BKs. Using ImageJ, a region of interest (ROI) of NxN pixels, where $N$ ranged from 7 to 9, was selected around individual bright spots and duplicated to a new window. A smaller ROI of $(\mathrm{N}-2) \times(\mathrm{N}-2)$ was drawn to capture all of the bright pixels in the duplicate window and the ImageJ function under Image $>$ Stacks $>$ Measure Stack was used to extract a time trace of the bright pixels, which is labeled as "PL-\#". In the same window, the ImageJ function under Edit>Selection>Make Inverse was used to select the surrounding background pixels and the same Measure Stack function was used to extract a time trace of the background pixels, which is labeled as "BG-\#". Each pair of PL and BG time traces are saved as .csv files and contain the following information (defined using Analyze>Set Measurements): Area, Standard Deviation, Min \& Max Gray Value, Mean Gray Value (a proxy for average intensity). Figures S14 and S15 show sample pairs of PL and BG traces of BKQDs and TAMRA-BKs.

A MATLAB (v. 2020A) file "Extract_photophys.mlx" containing a set of custom scripts was then used to analyze each pair PL and BG time traces for the BKQDs and TAMRA-BKs to calculate power law distributions, blink rate in blinks per second (bps), and SNR. The first script "getData.m" extracts the time series data from the csv files and performs: (1) background subtraction "PL-BG", defined as mean of the BG trace subtracted from the PL (2) a determination if the trace contains a cluster of emitters (3) generates output files. Traces that contain a cluster are defined by a broad distribution of intensity values in a probability distribution function fitted with a smoothing kernel. ${ }^{5,6} \mathrm{~A}$ peak fitting algorithm identifies the peak positions and widths of the distribution; a peak width greater than $10 \%$ of the bit depth ( 8 or 16 ) is labeled as a trace with multiple emitters (see Figure S16). Additionally, if only a single peak is fitted, the difference between the weighted mean and median values, also known as the skew, is computed to determine if the trace is mainly comprised of "high" or "low" PL states.

These results are then analyzed by the script "blink_cp2.m" where the PL trace is converted to a binary on-off trace. A changepoint trajectory is fitted to the "PL-BG" time trace to essentially denoise the data and prevent false on-off transitions near the threshold (Figure S17). The threshold "on" PL is defined by whether the trace contains a cluster and whether the trace has a well-defined "low" PL state from the previous analysis. If the trace contains a cluster and has a defined low state, then the threshold is defined as a standard deviation above the PL at the peak position of the low state. If the trace contains a single emitter and defined low state, then the threshold is defined as three times the standard deviation above the PL at the peak position of the low state. If the trace lacks a defined low state, regardless of number emitters, the threshold is defined as three times the standard deviation above the minimum value of the BG trace. Lastly, "computeSNR" and "MakeFitHistograms" output the average SNR and blink rate (Figures S18 \& S19). SNR was computed using eq 3 based on the pixel selection outline in Figure S13.

$$
S N R=\frac{\text { mean intensity of emitter ROI pixels }}{\text { standard deviation of background ROI pixels }}
$$




\section{SIMULATIONS OF EMITERS}

The analysis of individual traces BKQD and TAMRA-BK emitters resulted in a pair of values for SNR and blink rate for each fluorophore. Permutations of these paired values resulted in four distinct combinations of SNR and blink rate parameters that were used to create simulated emitters. The custom MATLAB script "SingleEmitter.m" creates a multi-frame movie of an individual emitter based on one of the four pairs of photophysical parameters. An ideal image of the point emitter is blurred by an aperture function to simulate a diffraction-limited spot and replicated $\mathrm{N}$ times to create a multi-frame movie, where $\mathrm{N}=1000$ or 3000 frames (Figure S20A). Each movie is then scaled to an SNR within the ranges extracted for CdSe/CdS QDs (Figure S18) and TAMRA (Figure S19). This is done by randomly scaling each pixel associated with the BG to a produce a desired standard deviation within the BG pixels. Then the pixels associated with the PL are randomly scaled to produce a desired mean intensity level Poisson-Gaussian noise is then introduced to the series of images using the shot noise and read noise values reported for the pco.edge $4.2 \mathrm{sCMOS}$ (Figure S2OB and C). Lastly, R randomly selected frames are set to an "off" state to simulate a "blink", where R represents the number of frames in a N-frame movie that need to "blink" to produce the desired blink rate parameter value (see Figure S20D and E for time traces extracted from simulated movies with the desired SNR and blink rate values). To create multi-frame simulations containing three overlapping emitter PSFs, the custom MATLAB script "MultiEmitter.m" was used. The same method was used to create a single emitter based on the pair of SNR and blink rate parameters. This individual emitter was then added to three different locations within a larger 11-by-11 pixel array to create a series of $\mathrm{N}$-images with well-overlapped emitters as diagrammed by Figure S21. All simulated movies are exported to a ".tif" format that can be read by ImageJ/FIJI using the "bfsave.m" script from the opensource BioFormats Toolbox for MATLAB downloaded from Open Microscopy Environment ${ }^{7}$.

\section{ANALYSIS OF SIMULATIONS}

Each simulation is then analyzed using the ImageJ plugin QuickPALM to produce an initial set of localized points. For single emitter simulations, these points are directly analyzed using the "Analysis_single.m" MATLAB script. The script bins the $X$ and $Y$ coordinates of the localized points from the QuickPALM output into histograms and fits them with a Gaussian (Figure S22). The peak location of the Gaussian represents to centroid coordinates and

the full-width half max (FWHM) of the Gaussian is related to the standard deviation by $s_{x, y}=\frac{F W H M_{x, y}}{2.354}$. For the multi-emitter simulations, the outputs from QuickPALM are processed using the "Analysis_multi.m" MATLAB script, where an hierarchical clustering algorithm is first applied to group the localized spots. A display the results of the cluster analysis for the user to then identify the specific clusters corresponding to one of the three simulated emitter locations (Figure S23). The points in the selected clusters are then binned to $X$ and $Y$ histograms and fitted with a Gaussian similar to what is done for the single emitter simulations (Figure S24). The results from each simulation are tabulated in Table S3 for the single-emitter movies and Tables S4 and S5 for the multi-emitter movies for a duration of 1000 and 3000 frames, respectively. For the multi-emitter analyses, there is a column for the QuickPALM analysis denotes the file name with associated input parameters and a column for the hierarchical clustering analysis denoting the method used ( $M=7$ max clusters, I = Inconsistency cutoff). 
Figure S1. Chemical structures of peptides used in this study.

A) Bradykinin, B) C-PEG-bradykinin, C) phosphatidylserine-tagged bradykinin, and D) TAMRA-tagged bradykinin.

A

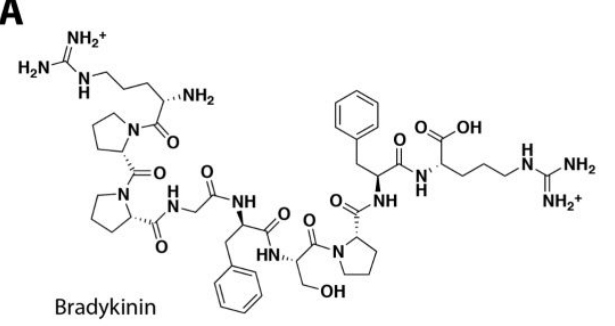

B

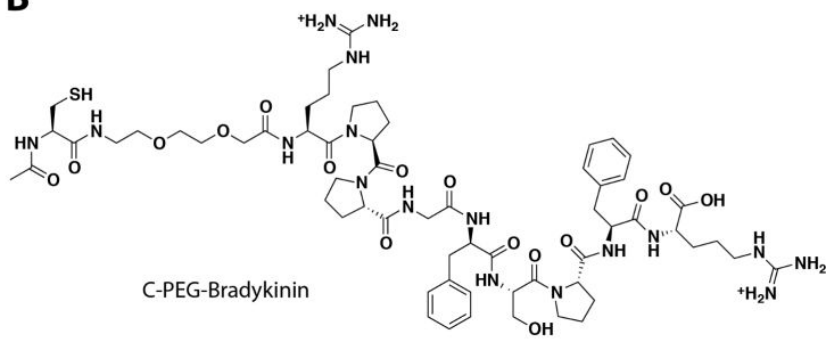

C

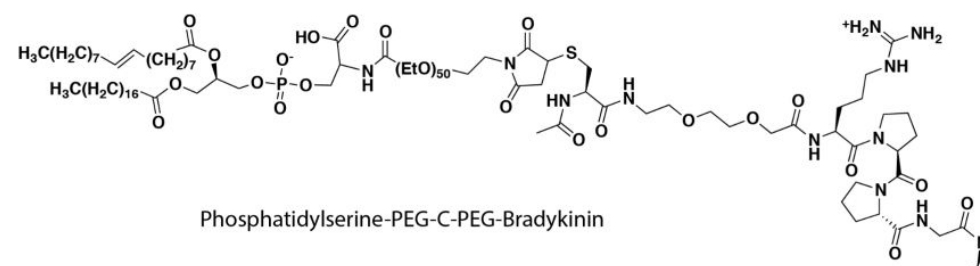

D

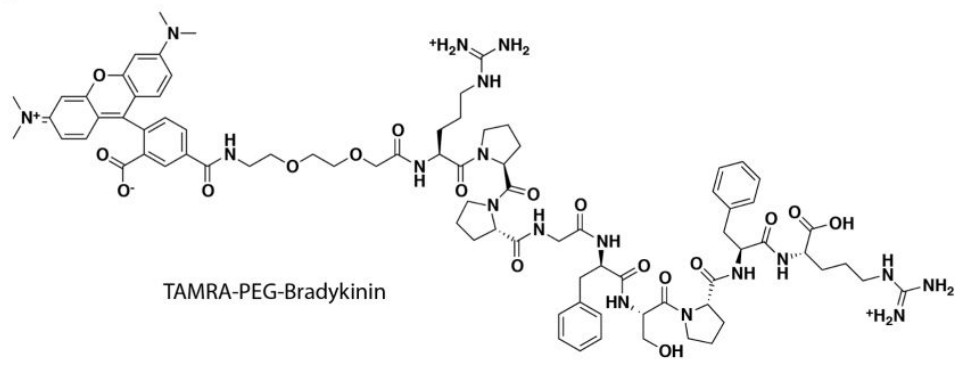

Figure S2. CdSe/CdS Characterization: Absorbance and Photoluminescence of CdSe Cores

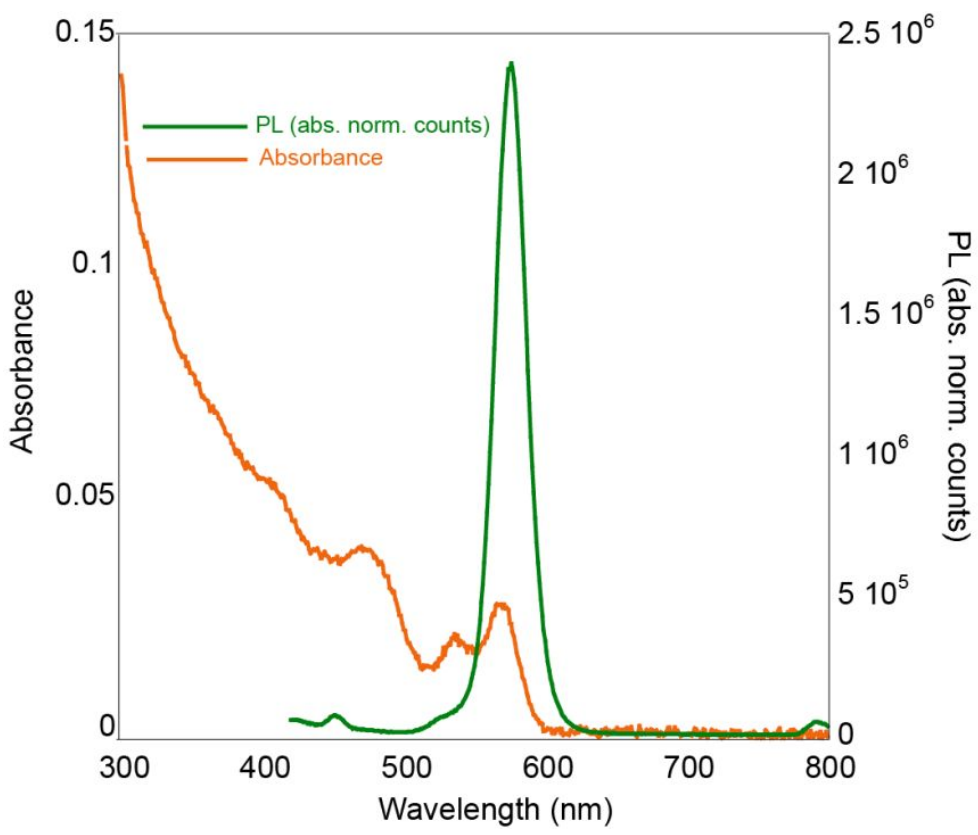


Figure S3. CdSe/CdS Characterization: Absorbance and Photoluminescence of CdSe/CdS Core/Shell QDs

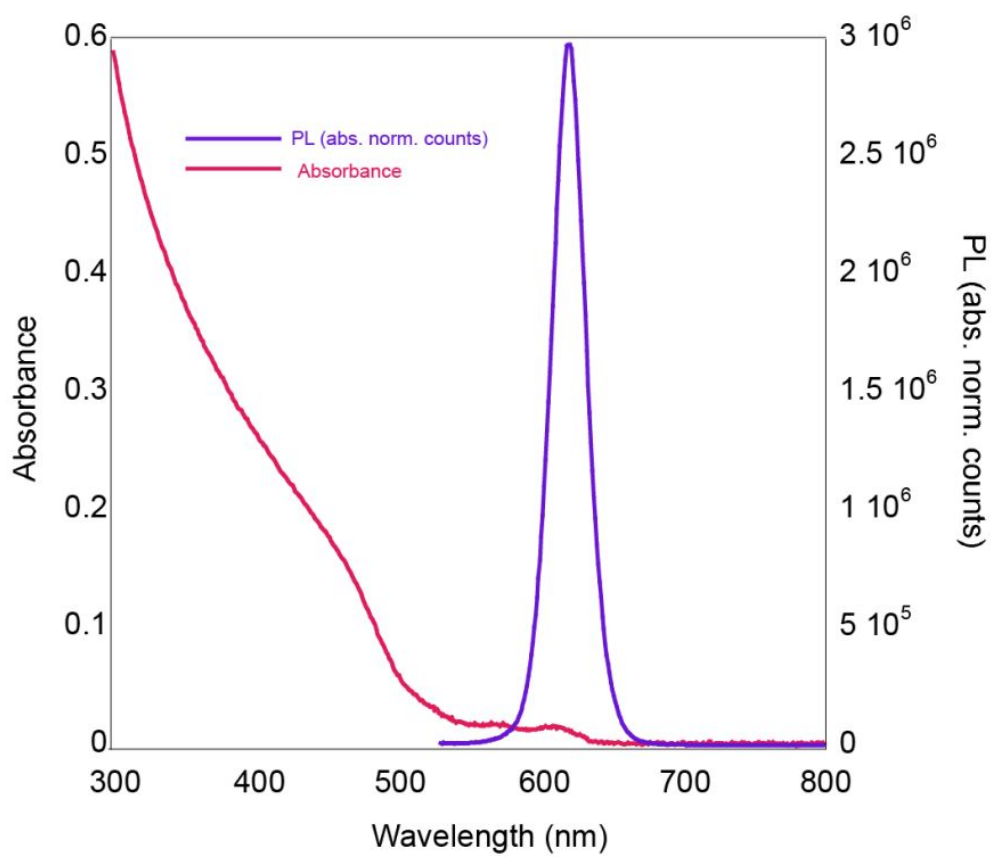

Figure S4. CdSe/CdS Characterization: TEM of CdSe/CdS Core/Shell QDs

Scale bars read represent $10 \mathrm{~nm}$ and $20 \mathrm{~nm}$ for the TEM image on the left and right, respectively.

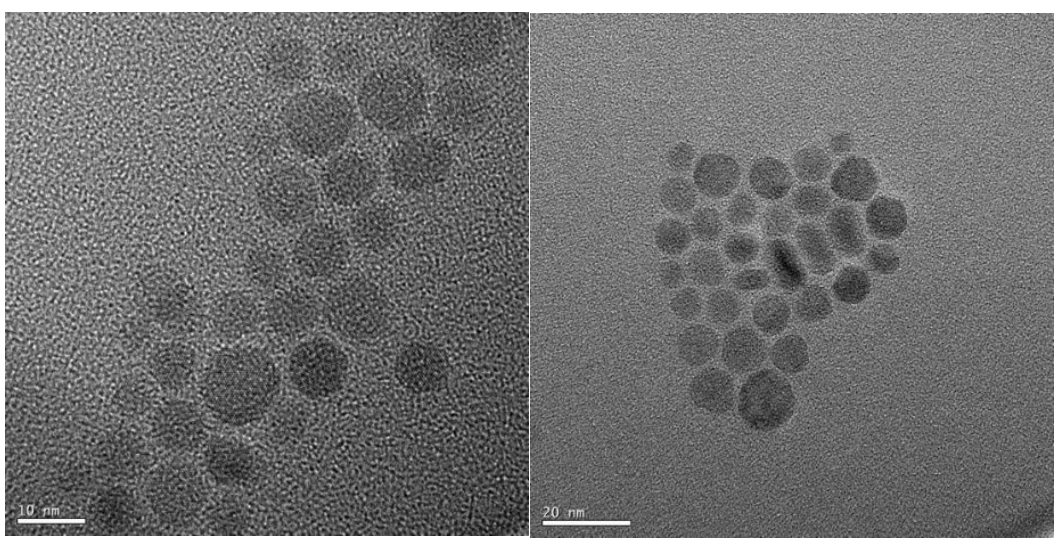

Table S1. Analytical HPLC gradient conditions

\begin{tabular}{|c|c|c|l|}
\hline Peptide & Sequence & $\begin{array}{c}\text { Retention } \\
\text { Time (min) }\end{array}$ & $\begin{array}{c}\text { Gradient (soln A: water/0.5\% TFA; } \\
\text { soln B: acetonitrile/0.5\% TFA) }\end{array}$ \\
\hline BK & RPPGFSPFR-OH & 11.15 & $\begin{array}{l}\text { Isocratic } 5 \% \text { B } 5 \mathrm{~min}, 5-95 \% \text { B over } 10 \mathrm{~min}, \\
95 \% \text { B } 5 \mathrm{~min}\end{array}$ \\
\hline C-PEG-BK & Ac-C-PEG-RPPGFSPFR-OH & 11.75 & $\begin{array}{l}\text { Isocratic } 5 \% \text { B } 5 \mathrm{~min}, 5-95 \% \text { B over } 10 \mathrm{~min}, \\
95 \% \text { B } 5 \mathrm{~min}\end{array}$ \\
\hline TAMRA-BK & TAMRA-PEG-RPPGFSPFR-OH & 12.30 & $\begin{array}{l}\text { Isocratic } 5 \% \text { B } 5 \mathrm{~min}, 5-95 \% \text { B over } 10 \mathrm{~min}, \\
95 \% \text { B } 5 \mathrm{~min}\end{array}$ \\
\hline
\end{tabular}


Figure S5. Analytical HPLC trace of synthetic peptide Bradykinin, BK, RPPGFSPFR-OH, at $215 \mathrm{~nm}$.

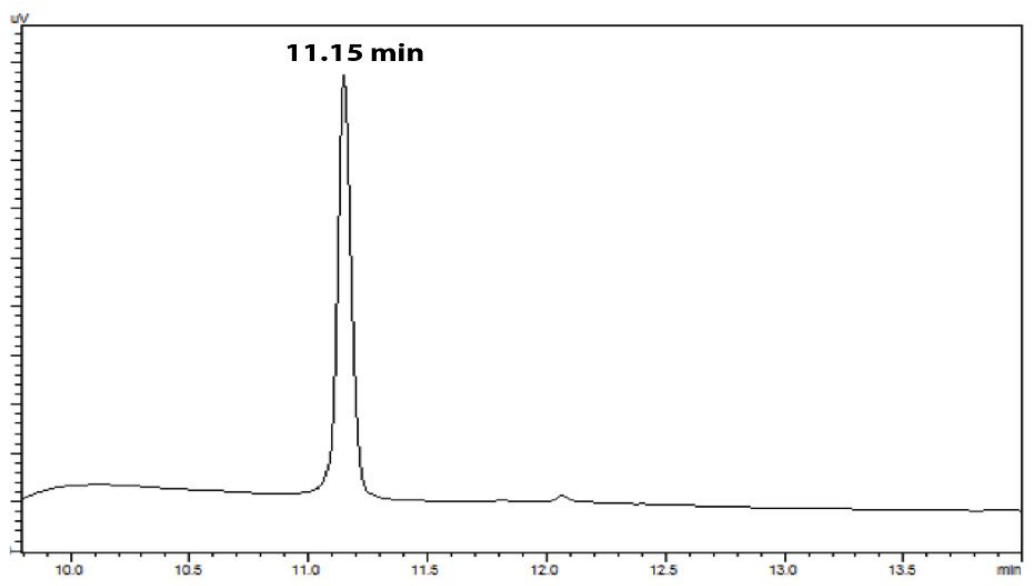

Figure S6. Analytical HPLC trace of synthetic peptide C-PEG-Bradykinin, C-PEG-BK, AC-C-PEG-RPPGFSPFR$\mathrm{OH}$, at $215 \mathrm{~nm}$

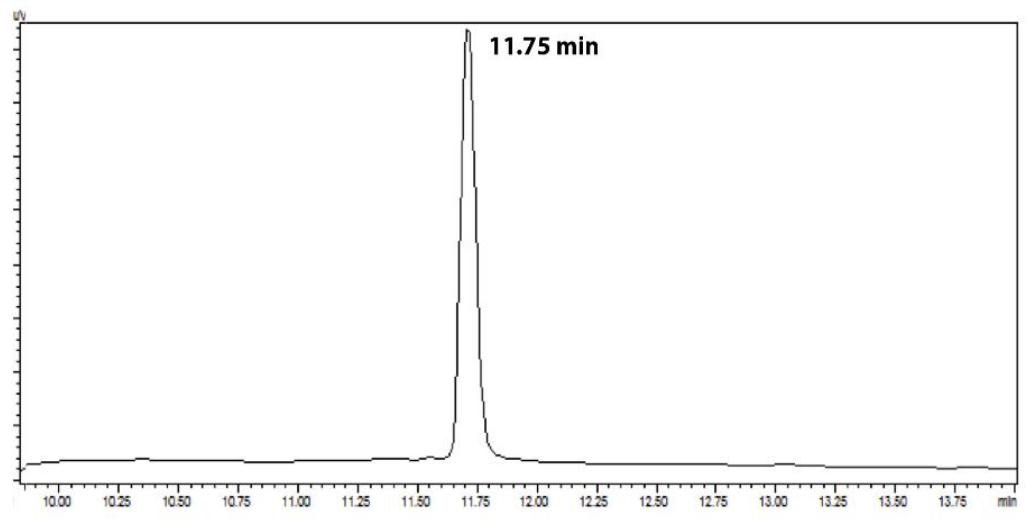

Figure S7. Analytical HPLC trace of synthetic peptide TAMRA-Bradykinin, TAMRA-BK, TAMRA-PEGRPPGFSPFR-OH, at $215 \mathrm{~nm}$

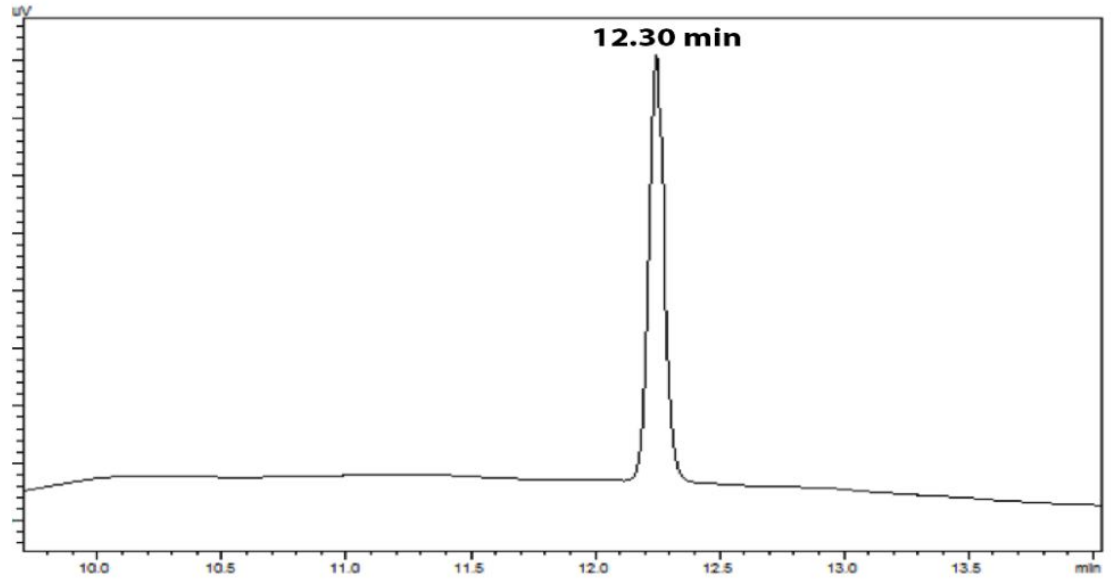


Figure S8. Peptide concentration curves used to determine concentrations of all peptides except TAMRABK (for which UV-Vis was used) in this study: Bradykinin, BK, RPPGFSPFR-OH

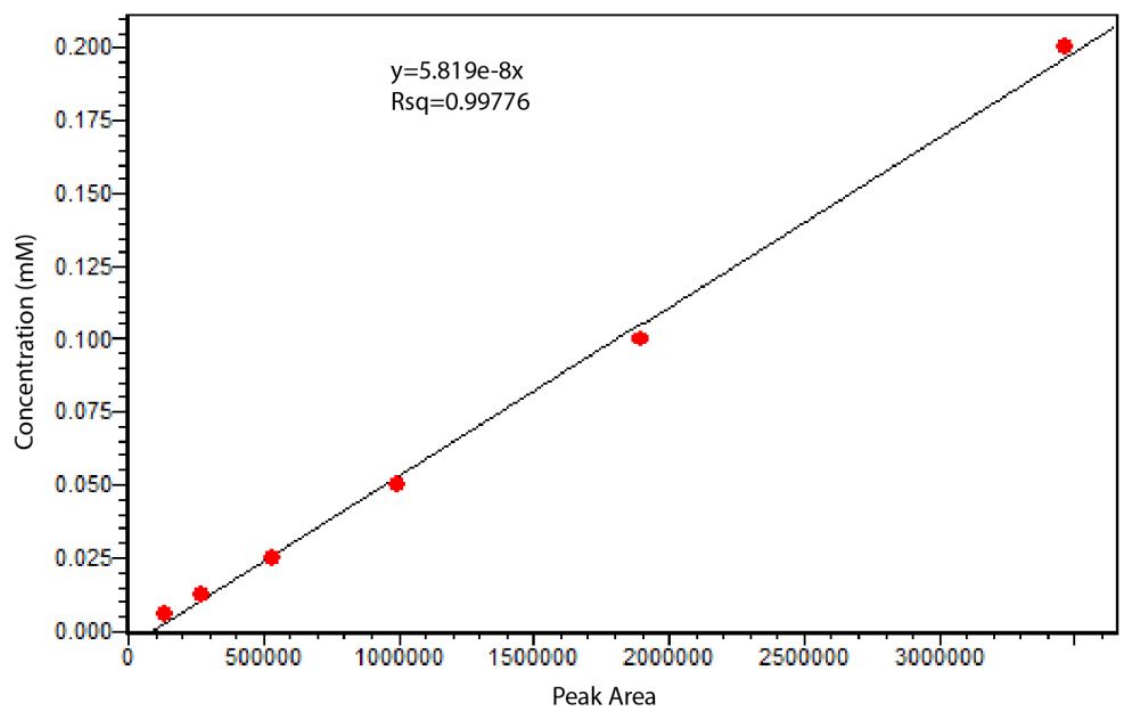

Figure S9. Peptide concentration curves used to determine concentrations of all peptides except TAMRABK (for which UV-Vis was used) in this study: C-PEG-Bradykinin, C-PEG-BK, Ac-C-PEG-RPPGFSPFR-OH

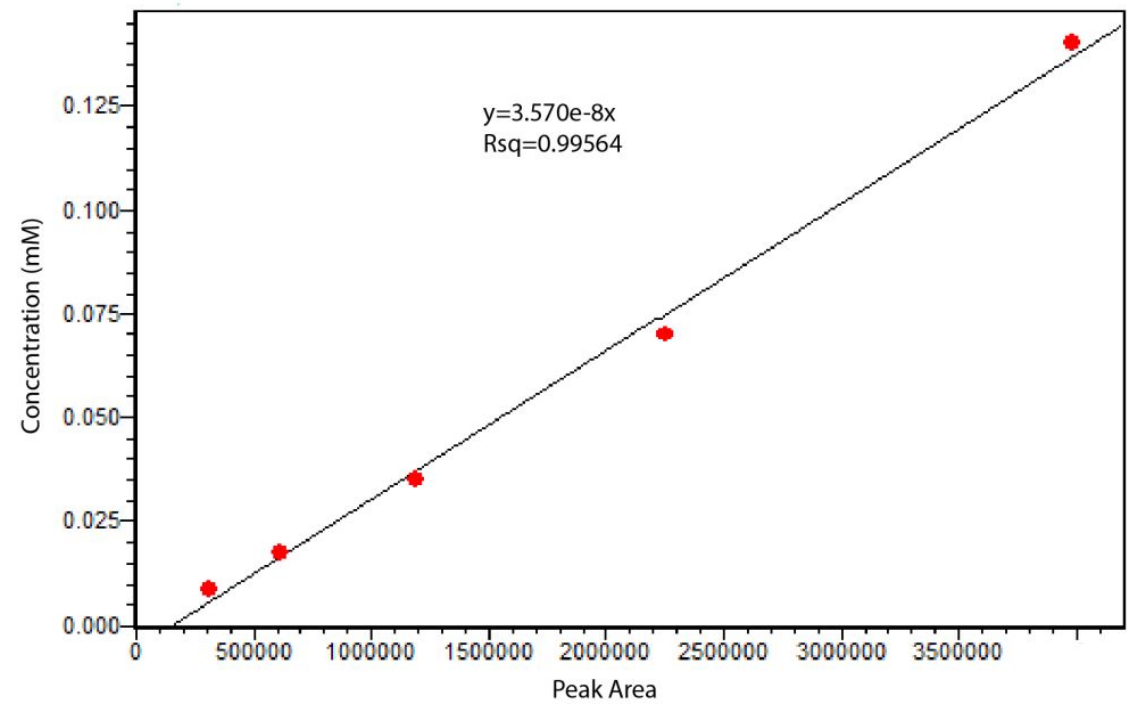

Table S2. Calculated and observed $\mathrm{m} / \mathrm{z}$ for all peptides by MALDI-TOF-MS.

\begin{tabular}{|c|c|c|}
\hline Peptide & calc $\left[\mathrm{MH}^{+}\right]$ & obs $\left[\mathrm{MH}^{+}\right]$ \\
\hline BK & 1061.58 & 1061.47 \\
\hline C-PEG-BK & 1351.67 & 1351.47 \\
\hline TAMRA-BK & 1618.79 & 1619.02 \\
\hline
\end{tabular}


Figure S10. MALDI-TOF-MS spectrum for Bradykinin, BK, RPPGFSPFR-OH

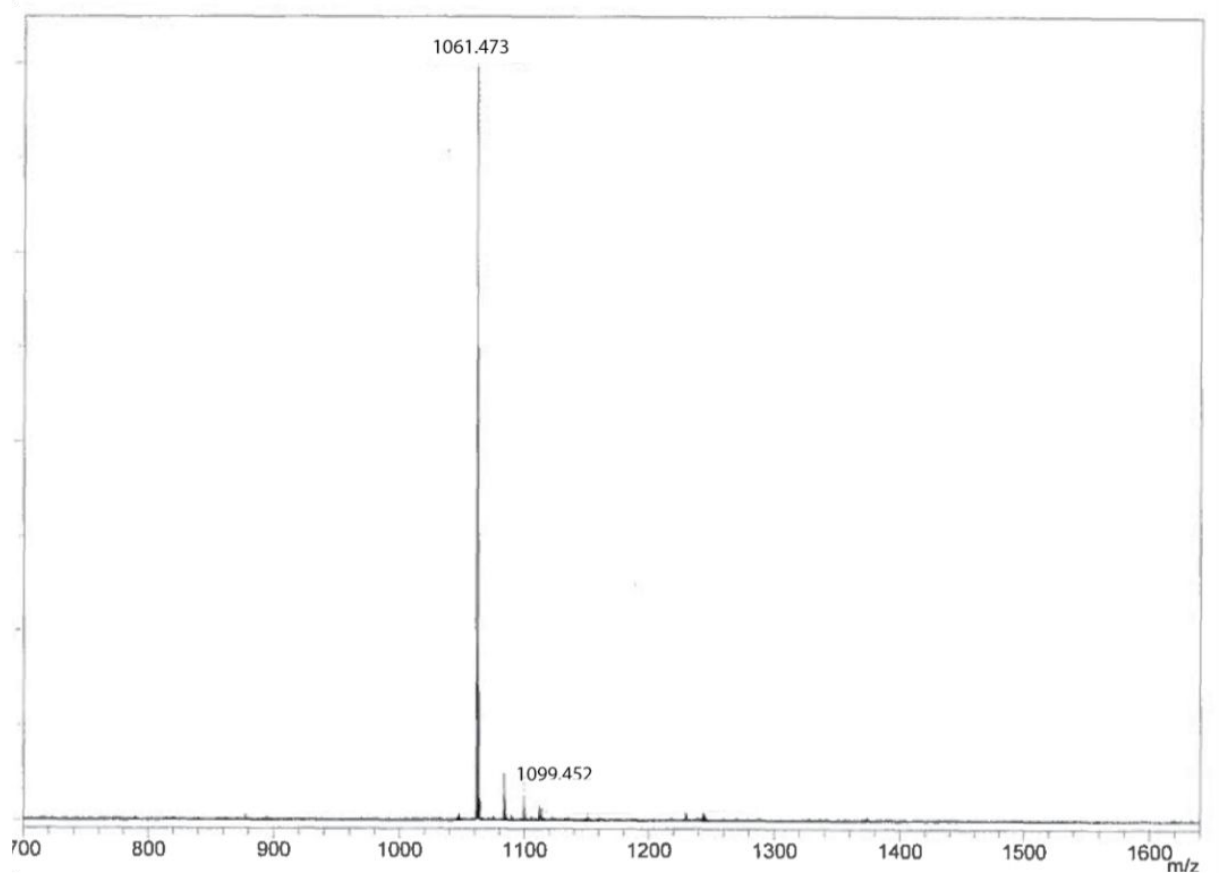

Figure S11. MALDI-TOF-MS spectrum for C-PEG-Bradykinin, C-PEG-BK, AC-C-PEG-RPPGFSPFR-OH

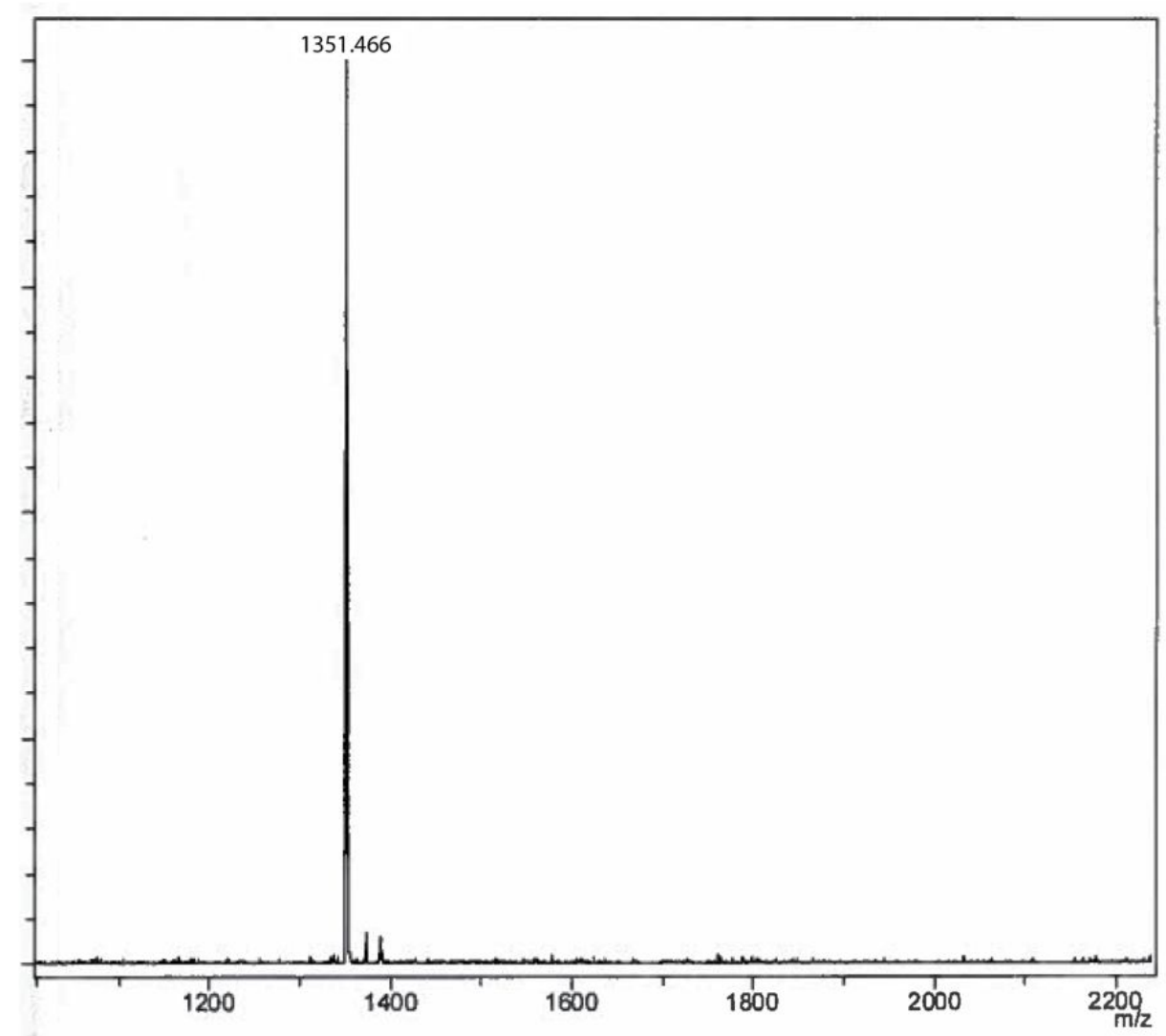


Figure S12. MALDI-TOF-MS spectrum for TAMRA-Bradykinin, TAMRA-BK, TAMRA-PEG-RPPGFSPFR-OH

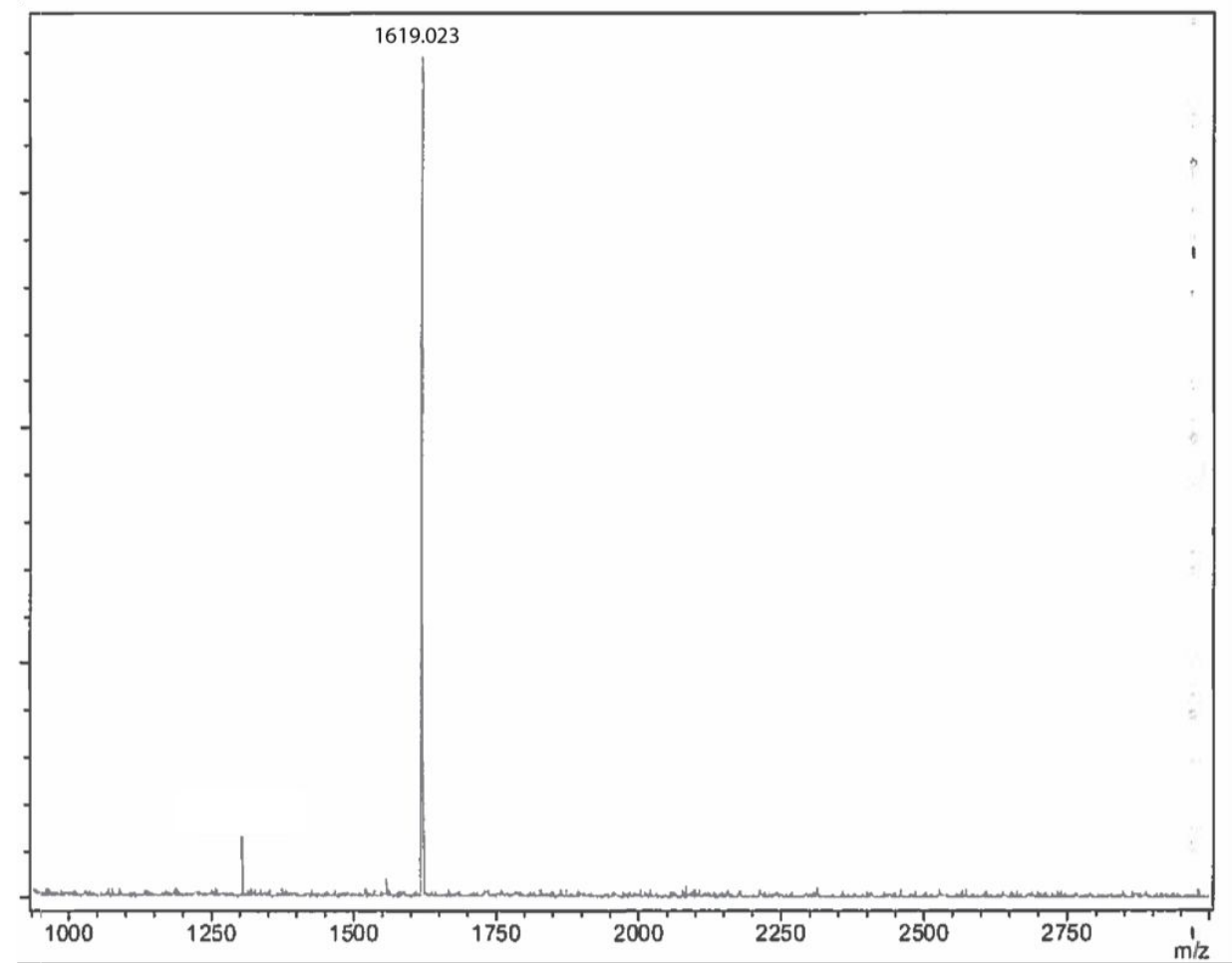

Figure S13. Diagram of pixel selection in ROI around bright, fluorescent spot.

The red circle represents the emitter, the shaded orange region including the emitter are denoted as the PL pixels, and the gray shaded region denotes the BG pixels.

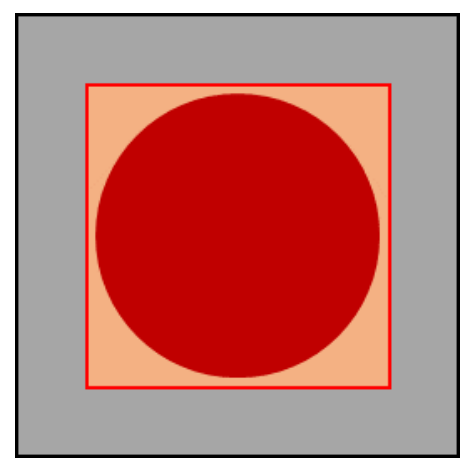

Figures S14. Representative time trace of BKQDs where the red-orange is the PL and the blue is the BG.

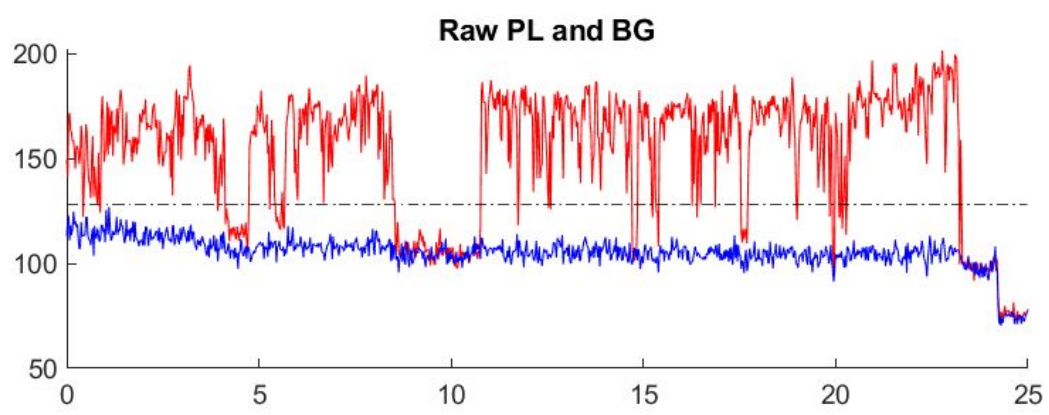


Figures S15. Representative time trace of TAMRA-BKs.

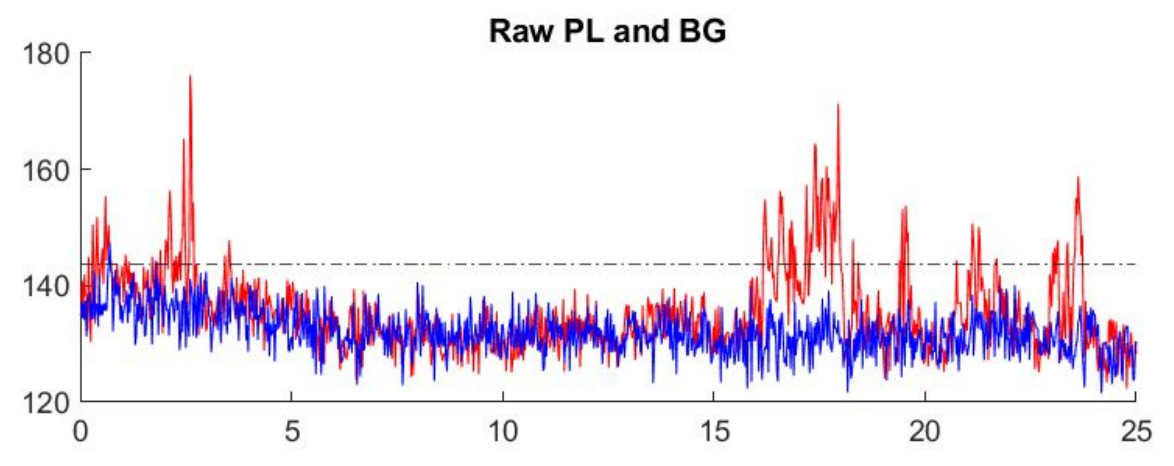

Figure S16. Background subtracted time trace, "PL-BG", and a smoothing kernel peak fit around the PDF of the PL intensity values.
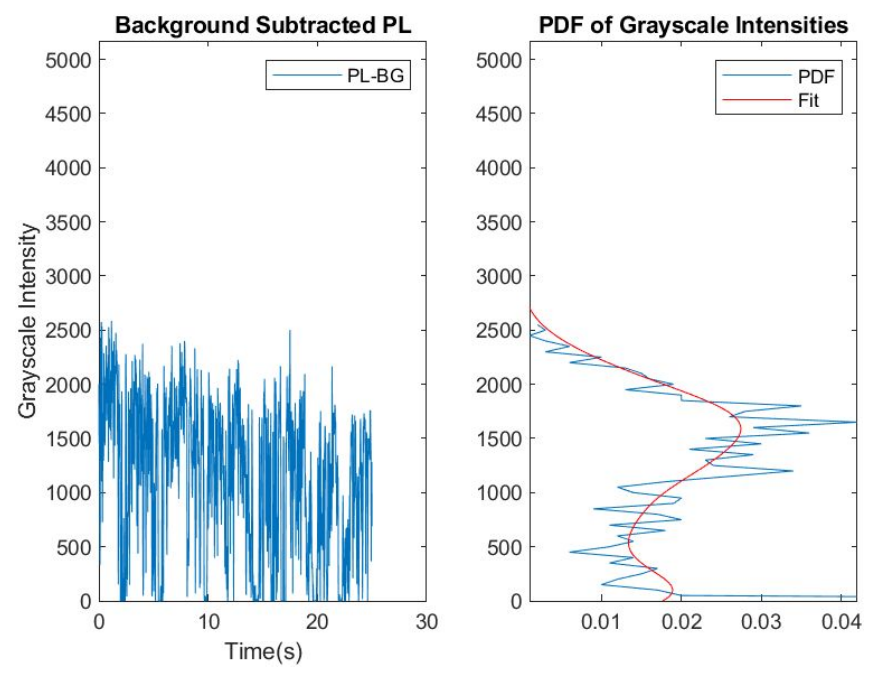

Figure S17. Changepoint trajectory (CP) fitted to "PL-BG" trace and subsequent "On-Off" transition blinking trace about the threshold.
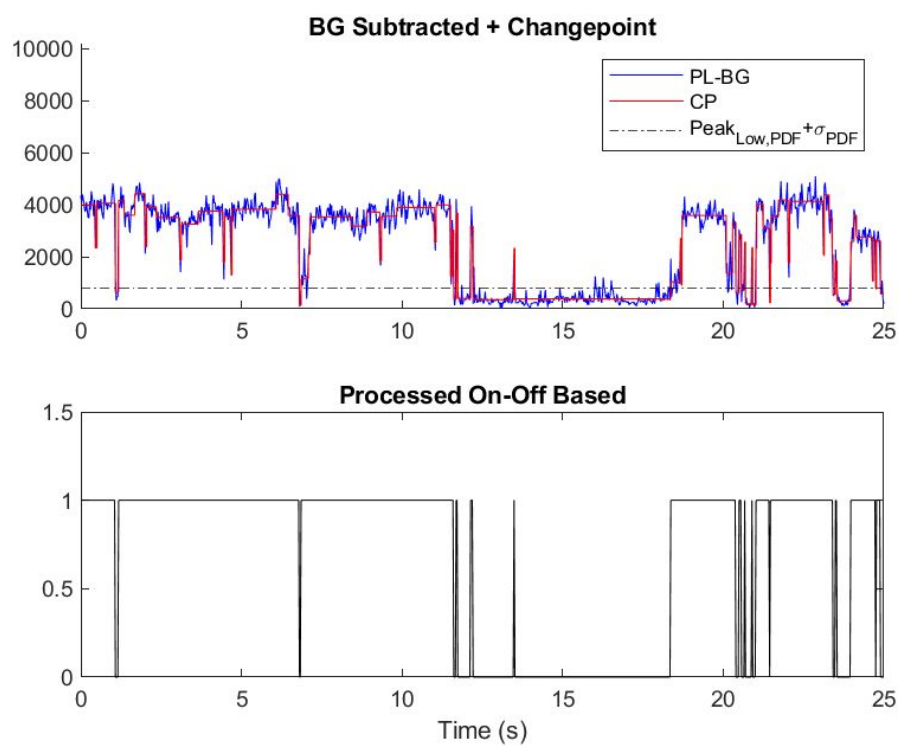
Figure S18. Histogram for SNR and blink rate of BKQDs.
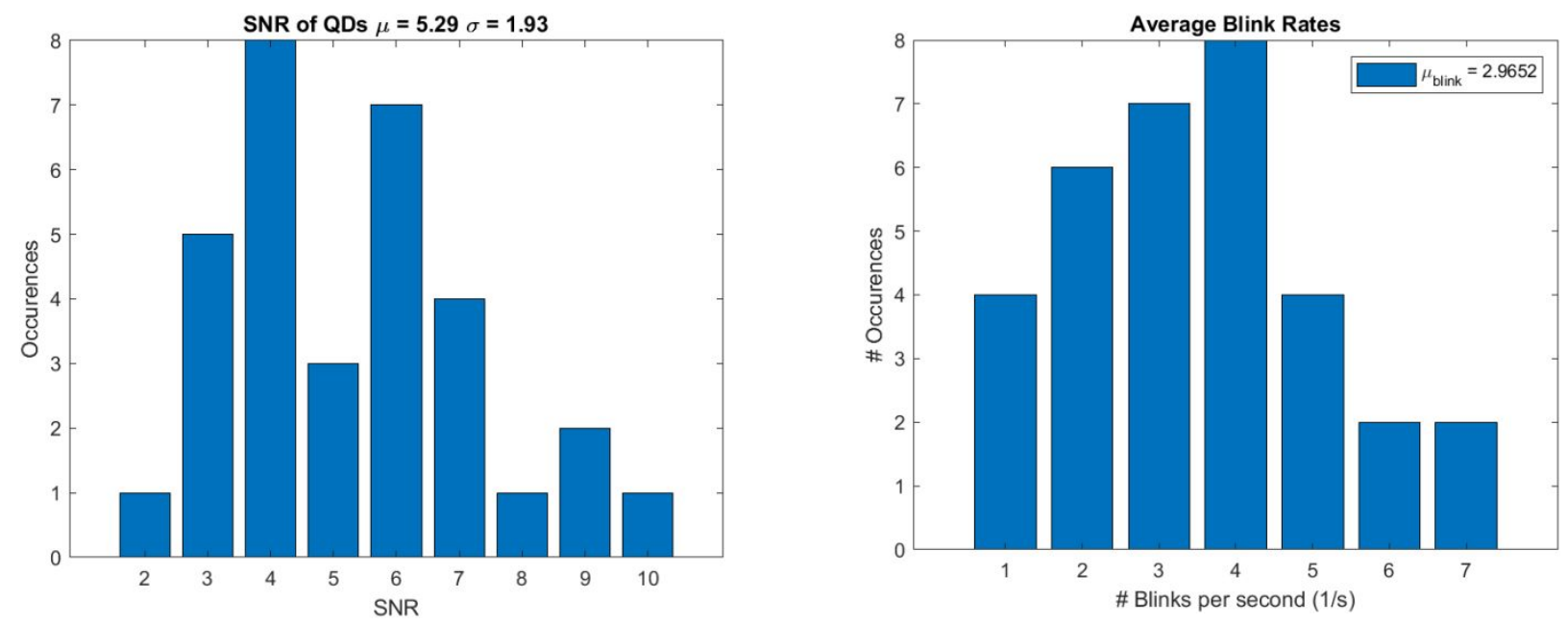

Figure S19. Histogram for SNR and blink rate of TAMRA-BKs.
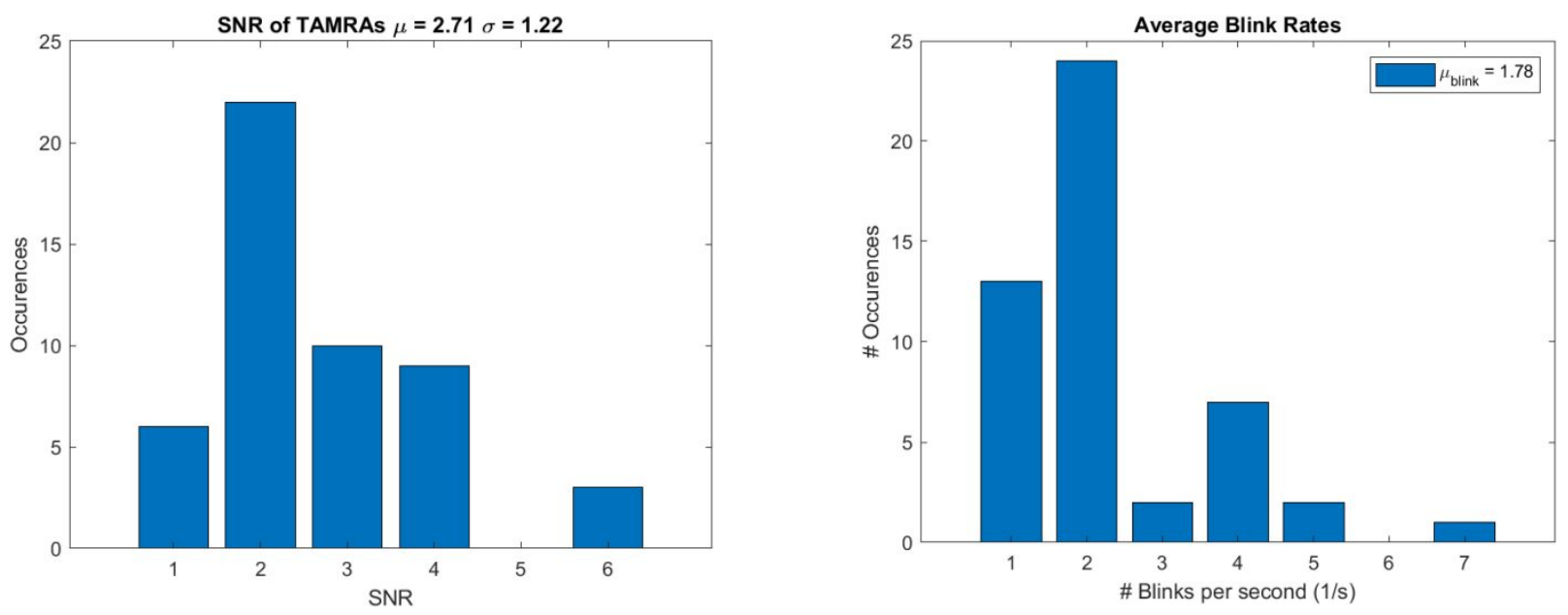
Figure S20. Creation of multi-frame movies of a simulated emitter.

A) A convolution between a simulated ideal emitter and aperture function produce a simulated diffraction-limited spot. B,C) Introduced Gaussian and Poisson, GP, noise alters each frame independently for the "low" and "high" SNR parameter simulations. D) Randomly selected frames are set to be "off" in both the "low" and "high" SNR simulations to achieve a blink rate of 2 blinks/sec. E) The same for 3 blinks/sec.
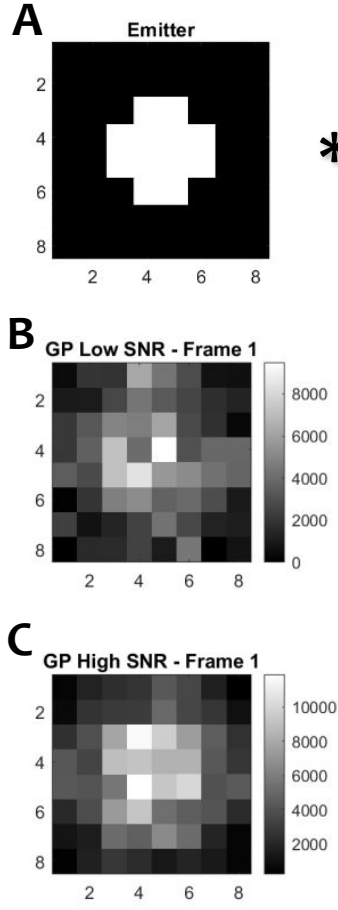
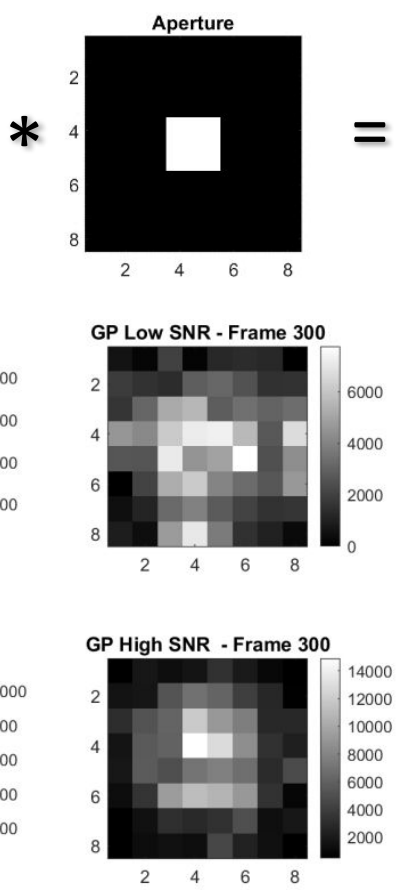
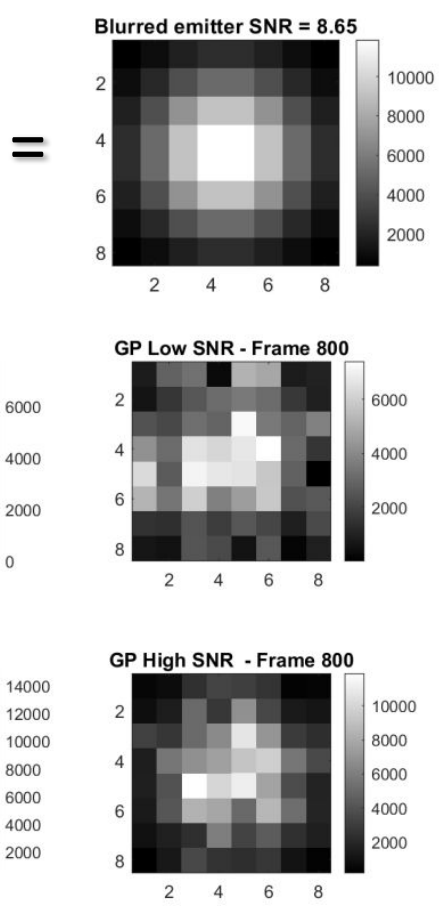

D
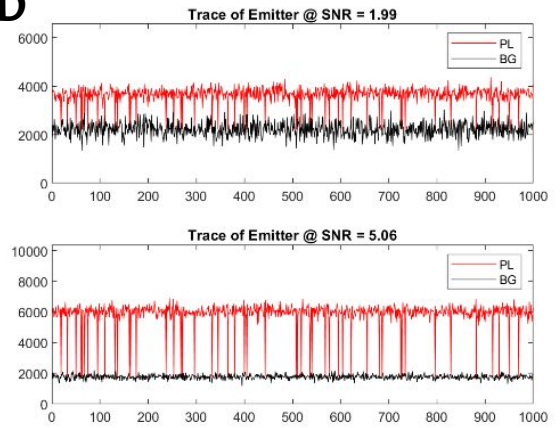

$\mathbf{E}$
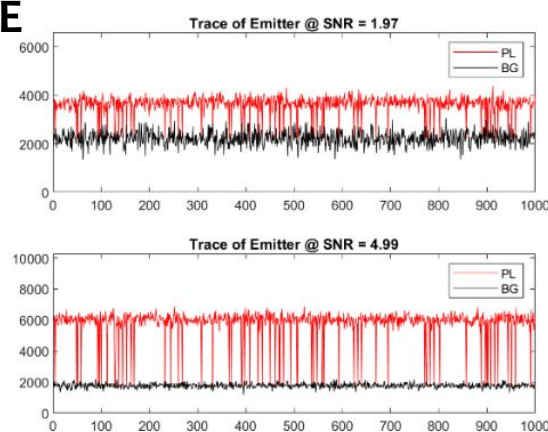

Figure S21. Diagram of how positions of the emitters are (A) defined in the multi-emitter simulations to ensure substantial overlap and (B) superimposed in the resultant simulation of three overlapped PSFs.

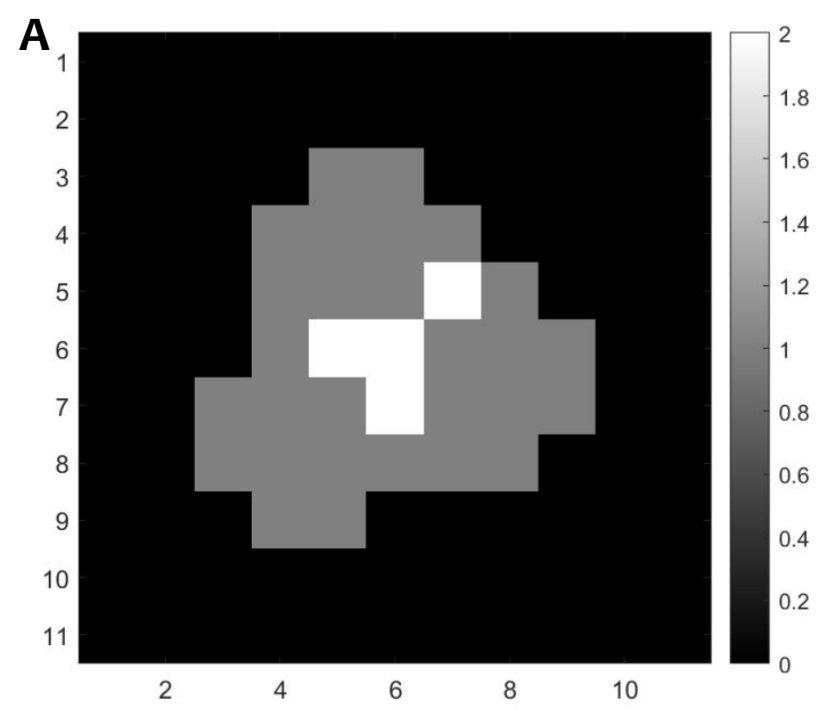

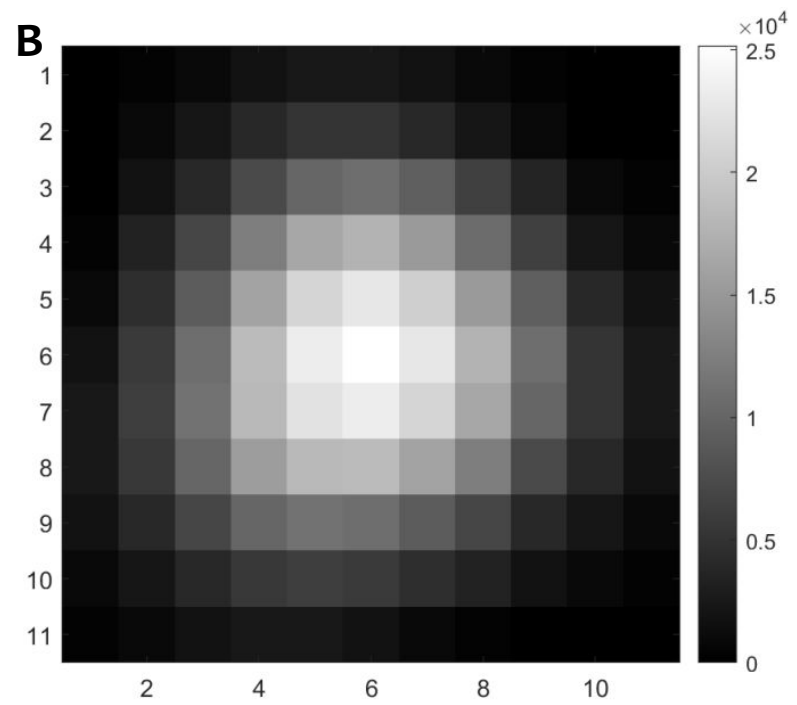


Figure S22. Gaussian fitted histograms for the $X$ and $Y$ coordinates of the localized points from QuickPALM analysis of single emitter simulations.

The peak position is the fitted centroid coordinates and the standard deviation is related to the $\frac{F W H M}{2.35}$.

Fitted Histograms for Centroid Location
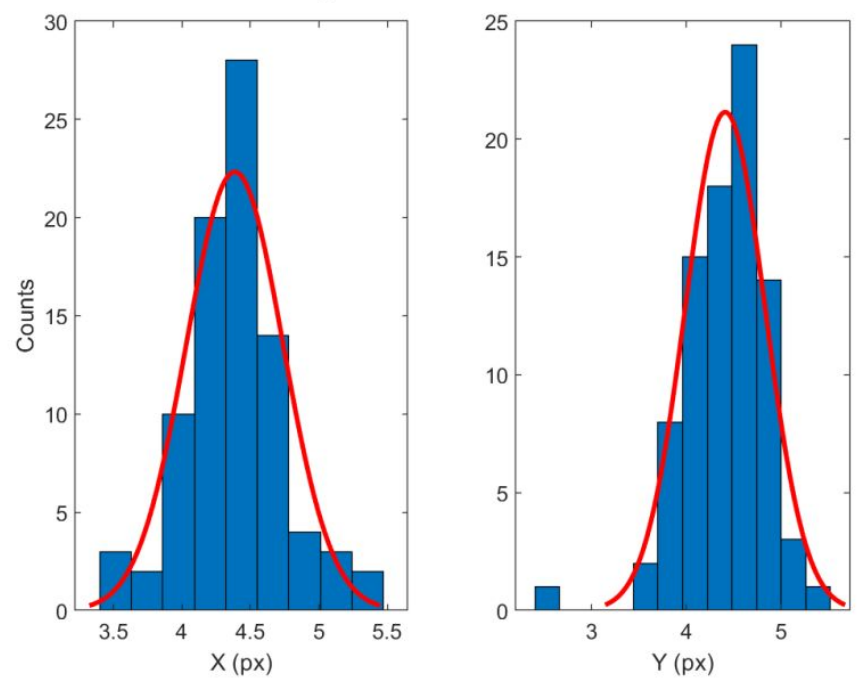

Figure S23. Example hierarchical clustering analysis of localized points from QuickPALM analysis of multiemitter simulations to identify groups of spots associated with one of the three simulated emitters.

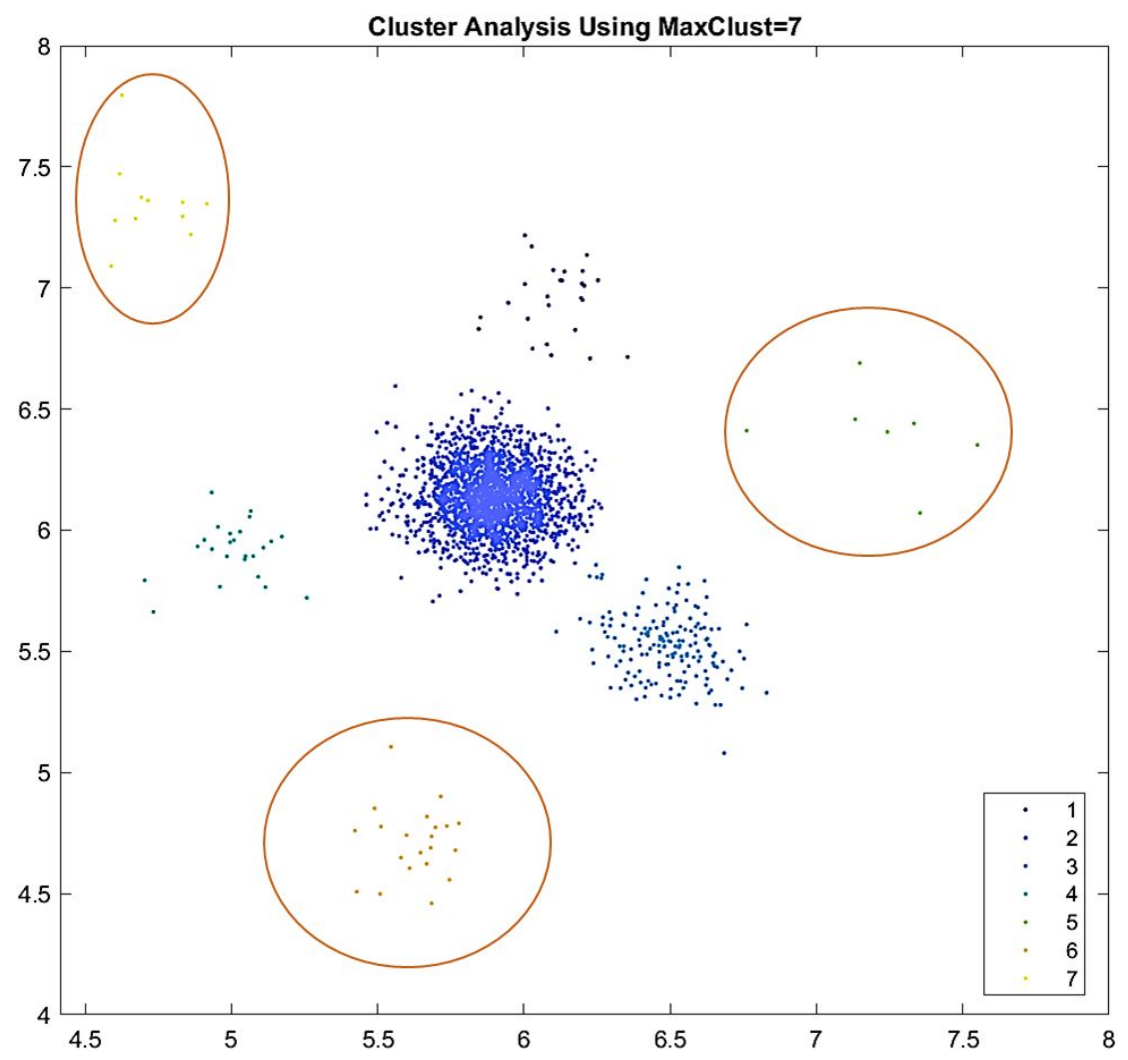


Figure S24. Gaussian fitted histograms for the $X$ and $Y$ coordinates of the localized points from QuickPALM analysis of overlapped multiple emitter simulations.

The peak position is the fitted centroid coordinates and the standard deviation is related to the $\frac{F W H M}{2.35}$.
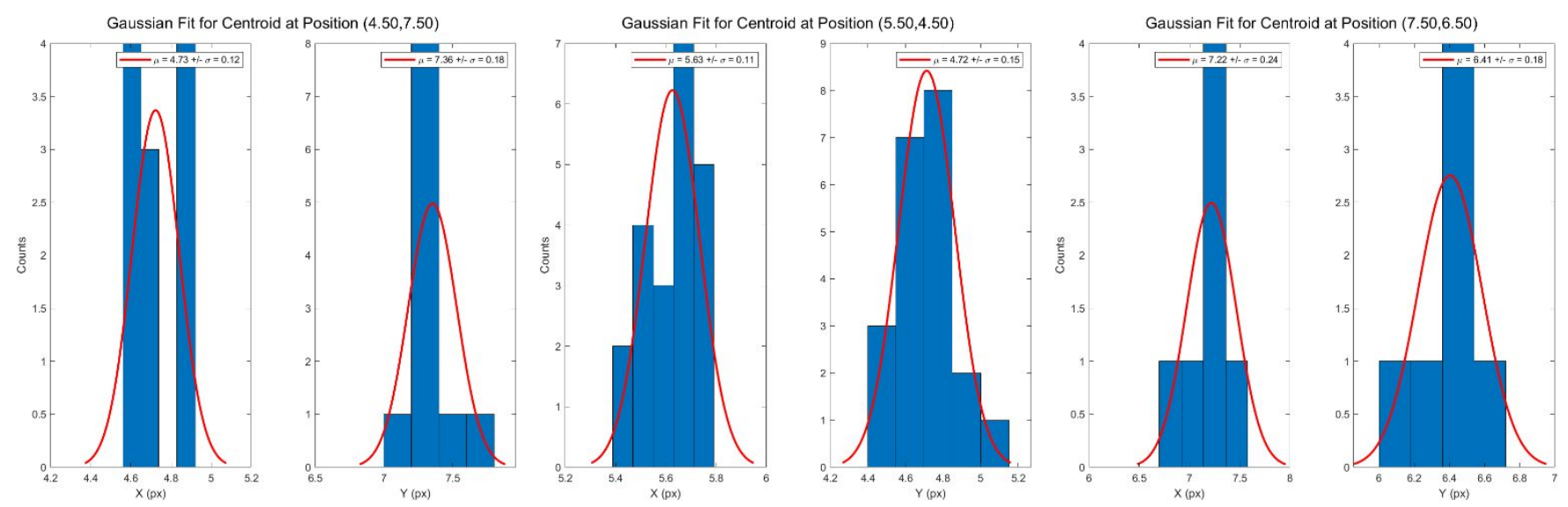

Table S3. Results of centroid localization analysis for $X$ and $Y$ coordinates of single emitter simulations

\begin{tabular}{|c|c|c|c|c|c|c|}
\hline Simulation & SNR & BPS & $\boldsymbol{\mu}_{\boldsymbol{X}}(\boldsymbol{p} \boldsymbol{x})$ & $\boldsymbol{\mu}_{\boldsymbol{y}}(\boldsymbol{p x})$ & $\boldsymbol{s}_{\boldsymbol{X}}$ & $\boldsymbol{s}_{\boldsymbol{y}}$ \\
\hline 1 & High & 2 & 4.4996 & 4.5076 & 0.0048 & 0.0048 \\
\hline 2 & High & 2 & 4.5089 & 4.4993 & 0.0047 & 0.0046 \\
\hline 3 & High & 2 & 4.5094 & 4.5012 & 0.0046 & 0.0046 \\
\hline 4 & Low & 2 & 4.4132 & 4.5316 & 0.0524 & 0.0172 \\
\hline 5 & Low & 2 & 4.4784 & 4.463 & 0.0039 & 0.004 \\
\hline 6 & Low & 2 & 4.504 & 4.5102 & 0.0041 & 0.0038 \\
\hline 7 & High & 3 & 4.4996 & 4.5077 & 0.0049 & 0.0048 \\
\hline 8 & High & 3 & 4.5089 & 4.4993 & 0.0047 & 0.0047 \\
\hline 9 & High & 3 & 4.5094 & 4.5012 & 0.0046 & 0.0046 \\
\hline 10 & Low & 3 & 4.4141 & 4.5316 & 0.0515 & 0.0172 \\
\hline 11 & Low & 3 & 4.4784 & 4.4628 & 0.0038 & 0.004 \\
\hline 12 & Low & 3 & 4.5042 & 4.5101 & 0.0039 & 0.0037 \\
\hline
\end{tabular}


Table S4. Results of centroid position analysis for 1000-frame multi-emitter simulations

\begin{tabular}{|c|c|c|c|c|c|c|c|}
\hline SNR & bps & QuickPALM & Cluster & $\boldsymbol{\mu}_{\boldsymbol{X}}(\boldsymbol{p} \boldsymbol{x})$ & $\boldsymbol{\mu}_{\boldsymbol{y}}(\boldsymbol{p} \boldsymbol{x})$ & $\boldsymbol{s}_{\boldsymbol{X}}$ & $\boldsymbol{s}_{\boldsymbol{y}}$ \\
\hline High & 2 & 2SNR-2FWHM-50Thresh.csv & $\mathrm{M}$ & $\mathrm{NaN}$ & $\mathrm{NaN}$ & $\mathrm{NaN}$ & $\mathrm{NaN}$ \\
\hline High & 2 & 2SNR-2FWHM-50Thresh.csv & $\mathrm{M}$ & 7.129 & 5.921 & 0.129 & 0.32 \\
\hline High & 2 & 2SNR-2FWHM-50Thresh.csv & $\mathrm{M}$ & 5.389 & 7.039 & 0.163 & 0.294 \\
\hline High & 3 & 2SNR-2FWHM-62Thresh.csv & $\mathrm{M}$ & 5.069 & 5.089 & 0.077 & 0.159 \\
\hline High & 3 & 2SNR-2FWHM-62Thresh.csv & $\mathrm{M}$ & 6.826 & 6.786 & 0.014 & 0.304 \\
\hline High & 3 & 2SNR-2FWHM-62Thresh.csv & $\mathrm{M}$ & 5.244 & 7.114 & 0.203 & 0.121 \\
\hline Low & 2 & 2SNR-2FWHM-50Thresh.csv & $\mathrm{M}$ & 5.985 & 5.004 & $\mathrm{NaN}$ & $\mathrm{NaN}$ \\
\hline Low & 2 & 2SNR-2FWHM-50Thresh.csv & $\mathrm{M}$ & 7.267 & 6.639 & 0.21 & 0.211 \\
\hline Low & 2 & 2SNR-2FWHM-50Thresh.csv & $\mathrm{M}$ & 4.842 & 7.115 & 0.273 & 0.166 \\
\hline Low & 3 & 2SNR-2FWHM-50Thresh.csv & $\mathrm{M}$ & 5.116 & 4.767 & $\mathrm{NaN}$ & $\mathrm{NaN}$ \\
\hline Low & 3 & 2SNR-2FWHM-50Thresh.csv & $\mathrm{M}$ & 7.427 & 6.459 & 0.166 & 0.086 \\
\hline Low & 3 & 2SNR-2FWHM-50Thresh.csv & $\mathrm{M}$ & 4.999 & 7.231 & 0.305 & 0.129 \\
\hline
\end{tabular}

Table S5. Results of centroid position analysis for 3000-frame multi-emitter simulations

\begin{tabular}{|c|c|c|c|c|c|c|c|}
\hline SNR & bps & QuickPALM & Cluster & $\boldsymbol{\mu}_{\boldsymbol{X}}(\boldsymbol{p} \boldsymbol{x})$ & $\boldsymbol{\mu}_{\boldsymbol{y}}(\boldsymbol{p} \boldsymbol{x})$ & $\boldsymbol{s}_{\boldsymbol{X}}$ & $\boldsymbol{s}_{\boldsymbol{y}}$ \\
\hline High & 2 & 3SNR-2FWHM-50Thresh.csv & $\mathrm{M}$ & $\mathrm{NaN}$ & $\mathrm{NaN}$ & NaN & NaN \\
\hline High & 2 & 3SNR-2FWHM-50Thresh.csv & $\mathrm{M}$ & 6.898 & 6.862 & 0.27 & 0.265 \\
\hline High & 2 & 3SNR-2FWHM-50Thresh.csv & $\mathrm{M}$ & 4.984 & 7.493 & 0.19 & 0.3 \\
\hline High & 3 & 2SNR-2FWHM-25Thresh.csv & $\mathrm{M}$ & 5.618 & 4.655 & $\mathrm{NaN}$ & $\mathrm{NaN}$ \\
\hline High & 3 & 2SNR-2FWHM-25Thresh.csv & $\mathrm{M}$ & 7.279 & 6.853 & 0.17 & 0.223 \\
\hline High & 3 & 2SNR-2FWHM-25Thresh.csv & $\mathrm{M}$ & 4.49 & 7.601 & 0.132 & 0.032 \\
\hline Low & 2 & 2SNR-2FWHM-50Thresh.csv & $\mathrm{I}$ & 4.885 & 4.983 & 0.188 & 0.219 \\
\hline Low & 2 & 2SNR-2FWHM-50Thresh.csv & $\mathrm{I}$ & 7.376 & 6.524 & 0.083 & 0.071 \\
\hline Low & 2 & 2SNR-2FWHM-50Thresh.csv & $\mathrm{I}$ & 4.715 & 7.154 & 0.157 & 0.055 \\
\hline Low & 3 & 2SNR-2FWHM-50Thresh.csv & $\mathrm{I}$ & 5.075 & 4.767 & 0.141 & 0.244 \\
\hline Low & 3 & 2SNR-2FWHM-50Thresh.csv & $\mathrm{I}$ & 7.282 & 6.595 & 0.094 & 0.064 \\
\hline Low & 3 & 2SNR-2FWHM-50Thresh.csv & $\mathrm{I}$ & 4.195 & 7.568 & 0.058 & 0.066 \\
\hline
\end{tabular}

Table S6. Comparison of QD photophysical properties to those reported in literature for well-performing, spectrally similar fluorophores normalized to TAMRA

\begin{tabular}{|c|c|c|c|c|c|c|c|}
\hline & $\lambda_{e x} / \lambda_{e m}$ & $\boldsymbol{\epsilon}\left(x 10^{5}\right)$ & QY & $\begin{array}{l}\text { Survival } \\
\text { Fraction }^{\text {a }}\end{array}$ & $\begin{array}{l}\text { Avg \# } \\
\text { Blink }^{\text {a }}\end{array}$ & $\begin{array}{c}\text { Normalized }^{c} \\
\text { Blink }\end{array}$ & $\begin{array}{c}\text { Normalized }^{\mathrm{c}} \\
\epsilon * Q Y\end{array}$ \\
\hline BKQD e & $600 / 620$ & $4.0-4.5$ & $0.2-0.3$ & $1^{b}$ & - & $2.9 / 1.8=1.6^{d}$ & $4.35-6.12$ \\
\hline TAMRA & $546 / 575$ & 0.9 & 0.2 & 0.85 & 10 & 1 & 1 \\
\hline Alexa 568 & $578 / 603$ & 0.9 & 0.69 & 0.58 & 7 & 0.7 & 3.29 \\
\hline Atto 565 & 563 / 592 & 1.2 & 0.9 & 0.17 & 4 & 0.4 & 5.89 \\
\hline Alexa 647 & 650 / 665 & 2.4 & 0.33 & 0.83 & 14 & 1.4 & 4.29 \\
\hline Atto $647 \mathrm{~N}$ & 644 / 669 & 1.5 & 0.65 & 0.24 & 9 & 0.9 & 5.29 \\
\hline \multicolumn{8}{|c|}{ a) $\quad$ Based on values in MEA buffer conditions from Dempsey et al. ${ }^{8}$} \\
\hline b) & \multicolumn{7}{|c|}{ Within timescale of observations, QDs are known to be stable/not bleach } \\
\hline c) & \multicolumn{7}{|c|}{ Normalized to TAMRA values given that it is the reference fluorophore } \\
\hline d) & \multicolumn{7}{|c|}{ Normalized BKQD average blink rate in blinks per second (bps) to the average of TAMRA-BK } \\
\hline e) & \multicolumn{7}{|c|}{ Range of values for QDs due to variations from synthesis batches. } \\
\hline
\end{tabular}


Figure S25. Super-resolution imaging results of 30 day old samples showing the stability of fixed neuron samples treated with BKQDs over time.

A) frame of a 1000 frame movie of BKQD labeled, fixed neurons after they were stored for one month, B) Superresolution reconstructed image from that same movie with $30 \mathrm{~nm}$ resolution and a 20 SNR threshold, C) Magnified image of the area in the yellow box in A, D) Magnified image of the area in the yellow box in C.
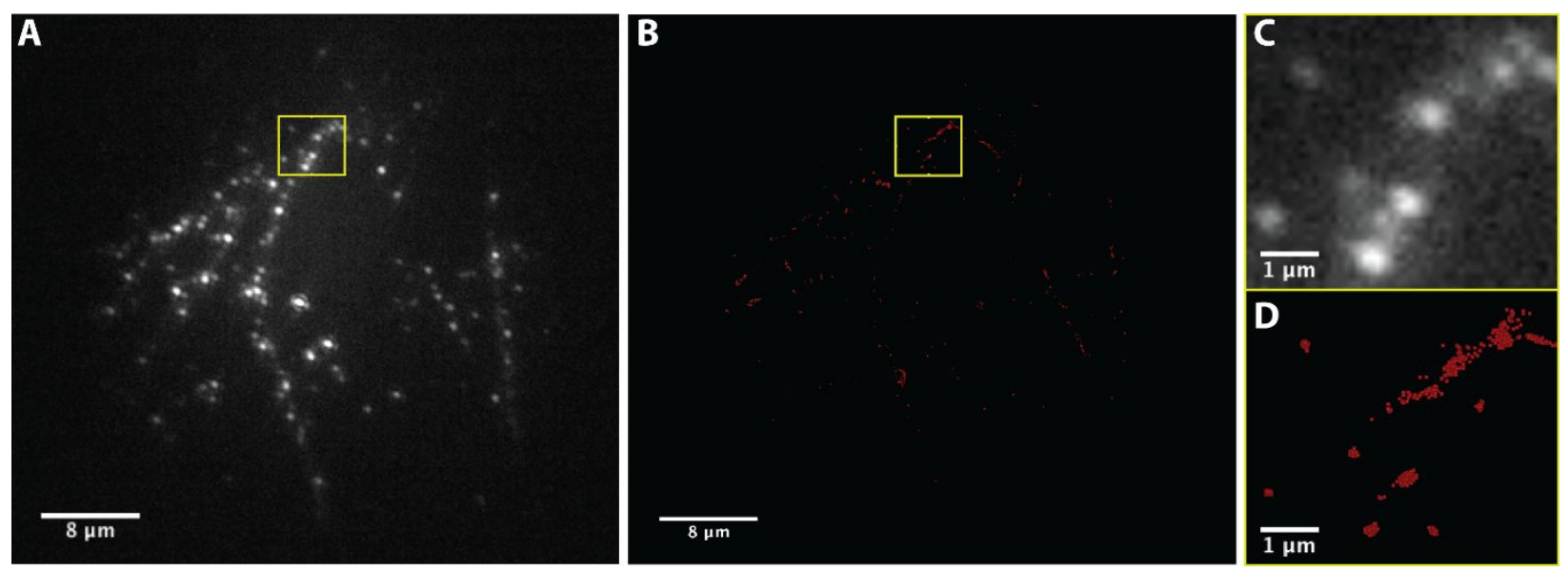

Figure S26. Super-resolution imaging results of 30 day old samples showing the instability of TAMRA-BK treated, fixed neuron samples over time.

A) First frame of a 1000 frame movie of TAMRA-BK labeled, fixed neurons after they were stored for one month, B) Super-resolution reconstructed image from that same movie with $50 \mathrm{~nm}$ resolution and a 6 SNR threshold, C) Magnified image of the area in the yellow box in A, D) Magnified image of the area in the yellow box in C.
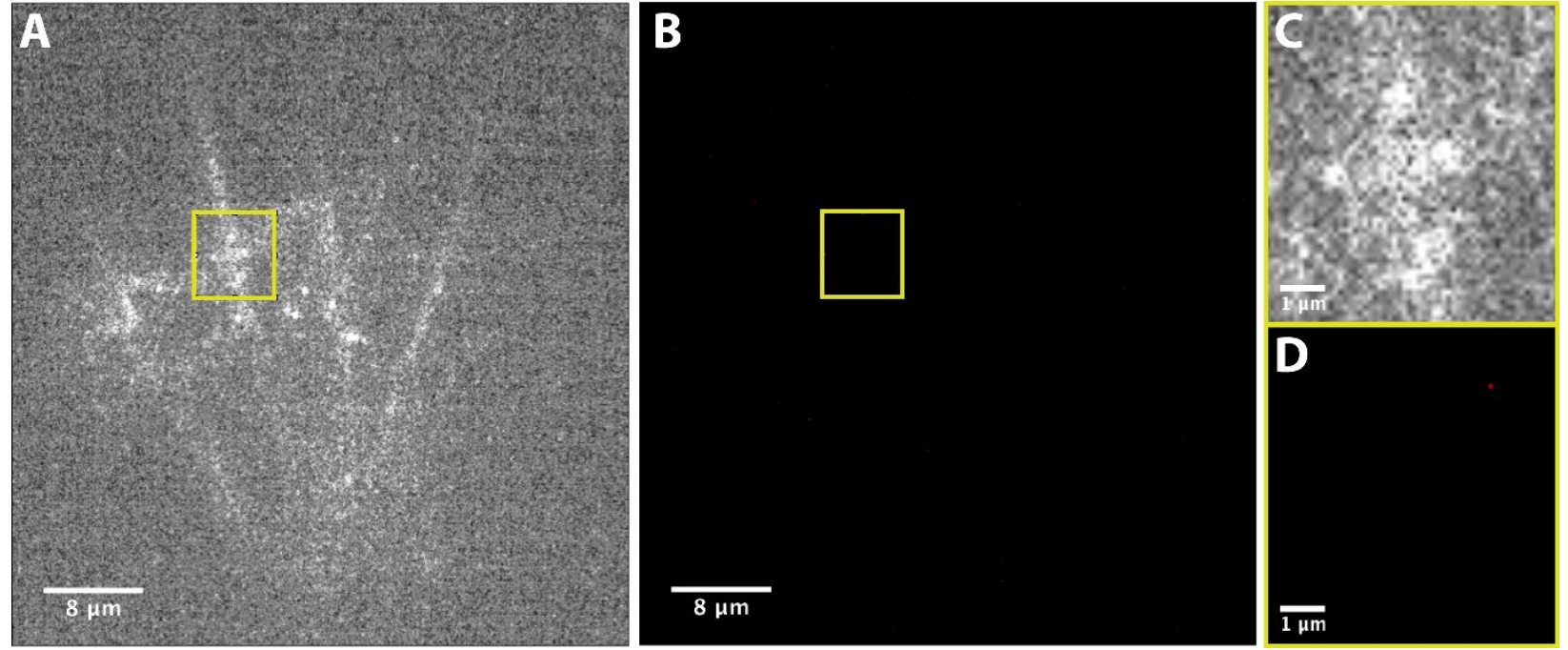
1. Kress, S. J.; Richner, P.; Jayanti, S. V.; Galliker, P.; Kim, D. K.; Poulikakos, D.; Norris, D. J., Near-field light design with colloidal quantum dots for photonics and plasmonics. Nano Lett 2014, 14 (10), 5827-33.

2. Maiseyeu, A.; Bagalkot, V., In vitro uptake of apoptotic body mimicking phosphatidylserine-quantum dot micelles by monocytic cell line. Nanoscale Res Lett 2014, 9 (1), 176.

3. Henriques, R.; Lelek, M.; Fornasiero, E. F.; Valtorta, F.; Zimmer, C.; Mhlanga, M. M., QuickPALM: 3D real-time photoactivation nanoscopy image processing in ImageJ. Nat Methods 2010, 7 (5), 339-40.

4. Wang, Y.; Fruhwirth, G.; Cai, E.; Ng, T.; Selvin, P. R., 3D super-resolution imaging with blinking quantum dots. Nano Lett 2013, 13 (11), 5233-41.

5.Ryan, D. P.; Goodwin, P. K.; Sheehan, C. J.; Whitcomb, K. J.; Gelfand, M. P.; Van Orden, A., Mapping Emission from Clusters of CdSe/ZnS Nanoparticles. J. Phys. Chem. C 2018, 122 (7), 4046-4053.

6. Whitcomb, K. J.; Ryan, D. P.; Gelfand, M. P.; Van Orden, A., Blinking Statistics of Small Clusters of Semiconductor Nanocrystals. J. Phys. Chem. C 2013, 117 (48), 25761-25768.

7. Linkert, M.; Rueden, C. T.; Allan, C.; Burel, J.-M.; Moore, W.; Patterson, A.; Loranger, B.; Moore, J.; Neves, C.; MacDonald, D., et al., Metadata matters: access to image data in the real world. Journal of Cell Biology 2010, $189(5), 777-782$.

8.Dempsey, G. T.; Vaughan, J. C.; Chen, K. H.; Bates, M.; Zhuang, X., Evaluation of fluorophores for optimal performance in localization-based super-resolution imaging. Nat Methods 2011, 8 (12), 1027-36. 